\title{
A PARABOLIC INVERSE PROBLEM WITH MIXED BOUNDARY DATA. STABILITY ESTIMATES FOR THE UNKNOWN BOUNDARY AND IMPEDANCE
}

\author{
V. BACCHELLI, M. DI CRISTO, E. SINCICH, AND S. VESSELLA
}

\begin{abstract}
We consider the problem of determining an unaccessible part of the boundary of a conductor by means of thermal measurements. We study a problem of corrosion where a Robin type condition is prescribed on the damaged part and we prove logarithmic stability estimate.
\end{abstract}

\section{INTRODUCTION}

In this paper we consider the inverse problem of determining an inaccessible portion $I$ of the boundary of a conducting body $\Omega \subset \mathbb{R}^{n}$ by means of thermal measurements, performed on an accessible part $A$ of its boundary. In particular, we analyze the situation when there might be a corrosion occurrence on $I$, modeled by an impedance type condition on the portion $I$ itself. The purpose of the inverse problem at hand is to recover information about both the unknown impedance and the unknown damaged part that, as it happens in practical contexts, cannot be directly inspected. Such an issue leads to the study of a parabolic equation with a Robin type condition on the inaccessible part of $\partial \Omega$ and a Dirichlet or Neumann condition, according to whether we prescribe a temperature or a heat flux, on $A$. This kind of boundary condition is known as a mixed type.

Assuming $\partial \Omega=\bar{A} \cup \bar{I}$ and $\operatorname{Int}_{\partial \Omega}(A) \cap \operatorname{Int}_{\partial \Omega}(I)=\emptyset$ and denoting by $\gamma(x, t)$ the surface impedance on $I$, we prescribe a heat flux $g$ on $A$ that induces a temperature $u$ in $\Omega$ solution to

$$
\begin{cases}u_{t}=\Delta u, & \text { in } \Omega \times[0, T], \\ u(x, 0)=0, & \text { in } \Omega, \\ \frac{\partial u}{\partial \nu}(x, t)=g(x, t), & \text { on } A \times[0, T], \\ \frac{\partial u}{\partial \nu}(x, t)+\gamma(x, t) u(x, t)=0, & \text { on } I \times[0, T],\end{cases}
$$

where $\nu$ is the outer unit normal to $\partial \Omega$.

The inverse problem we are addressing is to recover information about the unknown part $I$ of the boundary and on the impedance coefficient when thermal measurements of the form $\left\{g, u_{\mid \Sigma}\right\}$, where $\Sigma \subset A$, are available. Particularly, we are interested in the stability, i.e. the continuous dependence of $I$ and $\gamma$ from the boundary data.

Received by the editors September 1, 2011 and, in revised form, January 23, 2012. 2010 Mathematics Subject Classification. Primary 35R30, 35R25, 35R35. 
We remark that the mathematical model we consider here is a simplified version of the more general one in which the Robin condition is assumed on the whole boundary $\partial \Omega,[5,6,13$. In fact, our mixed boundary conditions correspond to the case in which the Biot number (a simple index of the ratio of heat transfer resistance inside of and at the surface of the body; see [25] Sect. 4.3.2, page 250) is very small (see also [5]). Nevertheless, it is rather simple to adapt our mathematical treatment to such a general situation (see Remark 4.6). At the same time the mixed condition permits us to simplify the exposition of the proof of our main result of the paper.

Under the assumption that $\gamma \equiv 0$ (i.e. the Neumann condition on an inaccessible portion $I$ ) the analysis of stability has been carried out both in the framework of elliptic and parabolic equations. In [2, 4, in which the problem is studied in the elliptic case, the authors show that, keeping as minimal as possible the a priori assumptions, the unknown boundary depends continuously on the known boundary measurements with a rate of continuity of logarithmic type, which is the best possible as shown in 9]. In the framework of parabolic equations, the stability issue has been studied in [8, 10, 23, 24]. Also, in such papers logarithmic stability estimates have been obtained. A refined analysis of the problem has been proposed in [10]. In such a paper the authors give a detailed study about the optimality of the logarithmic rate of continuity in the parabolic case.

Under the assumption that $\gamma \not \equiv 0$ the problem of determining the portion $I$ of the boundary and $\gamma$ has been considered in [3], where a uniqueness result in the stationary case is proved, provided two suitable measurements are performed. In view of the example given in [7], the number of measurements turns out to be optimal. The corresponding stability issue has been addressed in 22, where logarithmic stability estimates have been achieved. This result is optimal as well (see [9]). Let us finally mention [21, where a uniqueness result under weaker regularity assumptions on the boundary has been obtained. In the parabolic case the problem is considered in 14. In such a paper a uniqueness result, both for the Robin coefficient and the unknown portion $I$ of the boundary, is proved under the additional assumptions that $\gamma$ and $g$ do not depend on $t$ (more precisely, it is required that $g$ is time independent on an interval $\left.\left(T_{1}, T\right), T_{1}<T\right)$.

The main novelty of the present paper is to consider the case in which the Robin coefficient on the unknown boundary depends on $x$ and $t$. Such an assumption can be considered as a first step in studying the case in which $\gamma$ also depends on the temperature $u$. In this paper we show that $I$ depends on thermal boundary measurements on the accessible portion of the boundary with a rate of continuity of logarithmic type. Although the precise results are written in Theorem 2.5 below, here we would like to anticipate something about them and briefly discuss the approach we follow to prove them. As in the elliptic case, we perform two boundary measurements. More precisely we prescribe two different heat fluxes, $\widehat{g}$ and $\tilde{g}$, and we read the corresponding temperatures, $\widehat{u}$ and $\tilde{u}$, on a portion $\Sigma$ of the accessible part of the boundary. For $i=1,2$, let $\widehat{u}_{i}$ be the solution to (1.1) when $\Omega=\Omega_{i}$, $\gamma=\gamma_{i}, g=\widehat{g}$ and let $\tilde{u}_{i}$ be the solution to (1.1) when $\Omega=\Omega_{i}, \gamma=\gamma_{i}, g=\tilde{g}$. We assume that $\widehat{g}$ and $\tilde{g}$ are linearly independent and one of them with sign. We proved that if for a given $\varepsilon>0$ we have

$$
\begin{aligned}
\left\|\widehat{u}_{1}-\widehat{u}_{2}\right\|_{L^{2}(\Sigma \times(0, T))} & \leq \varepsilon, \\
\left\|\tilde{u}_{1}-\tilde{u}_{2}\right\|_{L^{2}(\Sigma \times(0, T))} & \leq \varepsilon,
\end{aligned}
$$


then

$$
d_{\mathcal{H}}\left(I_{1}, I_{2}\right) \leq C|\log \varepsilon|^{-\beta},
$$

where $d_{\mathcal{H}}\left(I_{1}, I_{2}\right)$ is the Hausdorff distance between $I_{1}$ and $I_{2}$ and $C$ and $\beta$ are positive constants that depend on the (a priori known) smoothness character of $\Omega_{i}$ and $\gamma_{i}$. Also a logarithmic stability estimate for the Robin coefficient holds true.

The main ideas and tools can be outlined as follows:

i) We introduce an auxiliary function $\lambda_{i}=\frac{\widehat{u}_{i}}{\tilde{u}_{i}}, i=1,2$ and we estimate how much the error $\varepsilon$ can affect the error on $\lambda_{1}-\lambda_{2}$. Such an estimate has been obtained by combining two arguments. The first relies on smallness propagation estimates based on an iterated use of two-sphere and onecylinder inequality $([11,24])$. The latter is a lower bound for the solutions $u_{i}$ achieved by combining the Harnack inequality up to the inaccessible boundary (see Proposition 4.3) with an iterated application of the interior Harnack inequality $(19,20])$.

ii) A lower bound for $\lambda_{i}$ which has been established by the use of quantitative estimates of unique continuation and a proper choice of the given heat fluxes. Precisely, we also prescribe a quantitative control of the linear independence of $\widehat{g}$ and $\tilde{g}$ described above.

iii) Using i) and ii) we prove a first rough estimate of log-log type for the Hausdorff distance between the unknown domains. Then, employing in a more refined way the above-mentioned estimates, in particular using the two-sphere one-cylinder inequality at the boundary (see Theorem 3.7 and [11,24]) and a geometric argument, we get the logarithmic estimate. The stability for the unknown impedance follows from stability estimates for the underlying Cauchy problem and the stability result for the unknown boundary.

For the sake of exposition, we have chosen to study the inverse problem for the constant coefficients equation. All proofs, though, can be simply adapted to the equation with coefficients depending on time and space with reasonable assumptions on them. Indeed, we deal with the auxiliary function $\lambda_{i}$ that solves an equation with variable coefficients.

The plan of the paper is the following. The main result is stated in Section 2 , where we also give notation and definitions. In Section 3 we provide a proof of this result based on some auxiliary propositions proved in the subsequent Sections 4, 5.

\section{MAIN RESUlT}

We begin by giving some notation and definitions. For every $x \in \mathbb{R}^{n}, n \geq 2$, $x=\left(x_{1}, \ldots, x_{n}\right)$, we set $x=\left(x^{\prime}, x_{n}\right)$, where $x^{\prime} \in \mathbb{R}^{n-1}$ and $x_{n} \in \mathbb{R}$. We denote by $B_{r}(x)$ and $B_{r}^{\prime}\left(x^{\prime}\right)$ respectively the open ball in $\mathbb{R}^{n}$ centered at $x$ of radius $r$ and the open ball in $\mathbb{R}^{n-1}$ centered at $x^{\prime}$ of radius $r$. Sometimes we shall write $B_{r}$ and $B_{r}^{\prime}$ instead of $B_{r}(0)$ and $B_{r}^{\prime}(0)$ respectively. For given numbers $r, t>0$ and a function $\varphi$ defined on $\mathbb{R}^{n-1} \times \mathbb{R}$, we define $\Omega_{r}=\{x \in \Omega$ : $\operatorname{dist}(x, \partial \Omega)>r\}, Q_{r, \varphi}^{t}=\{(x, s) \in$ $\left.B_{r} \times\left(t-r^{2}, t\right): x_{n}>\varphi\left(x^{\prime}\right)\right\}$ and $\Gamma_{r, \varphi}^{t}=\left\{(x, s) \in B_{r}^{\prime} \times\left(t-r^{2}, t\right): x_{n}=\varphi\left(x^{\prime}\right)\right\}$.

Denoting by $D$ an open subset of $\mathbb{R}^{n+1}$, for a function $f$ defined on $D$ and $\alpha \in(0,1]$, we define

$$
[f]_{\alpha ; D}=\sup \left\{\frac{|f(x, t)-f(y, s)|}{\left(|x-y|^{2}+|t-s|\right)^{\alpha / 2}}:(x, t),(y, s) \in D,(x, t) \neq(y, s)\right\} .
$$


If $\alpha \in(0,2]$, we set

$$
\langle f\rangle_{\alpha ; D}=\sup \left\{\frac{|f(x, t)-f(y, s)|}{|t-s|^{\alpha / 2}}:(x, t),(y, s) \in D, t \neq s\right\} .
$$

Let $k$ be a positive integer, $D$ an open subset of $\mathbb{R}^{n+1}, f$ a sufficiently smooth function and $\alpha \in(0,1]$. We denote this by

$$
\begin{aligned}
{[f]_{k+\alpha ; D} } & =\sum_{|\beta|+2 j=k}\left[\partial_{x}^{\beta} \partial_{t}^{j} f\right]_{\alpha ; D}, \\
\langle f\rangle_{k+\alpha ; D} & =\sum_{|\beta|+2 j=k-1}\left\langle\partial_{x}^{\beta} \partial_{t}^{j} f\right\rangle_{1+\alpha ; D},
\end{aligned}
$$

where for a multi-index $\beta=\left(\beta_{1}, \ldots, \beta_{n}\right), \beta_{i} \in \mathbb{N} \cup\{0\}, i=1, \ldots, n$, we have used the notation $\partial_{x}^{\beta} \partial_{t}^{j} f=\frac{\partial^{|\beta|+k} f}{\partial_{x_{1}}^{\beta_{1}} \ldots \partial_{x}^{\beta n} \partial_{t}^{k}}$, with $|\beta|=\sum_{i=1}^{n} \beta_{i}$. If $\alpha \in(0,1]$ and $[f]_{\alpha ; D}$ is finite, we shall say that $f$ belongs to $C^{0, \alpha}(D)$. Let $k$ be a positive integer, $\alpha \in(0,1]$ and $D$ an open subset of $\mathbb{R}^{n+1}$. We shall say that $f$ belongs to the class $C^{k, \alpha}(D)$ whenever for every non-negative integer $j$ such that $|\beta|+2 j \leq k$, there exist the derivatives $\partial_{x}^{\beta} \partial_{t}^{j} f$ and the quantities $\sup _{D}\left|\partial_{x}^{\beta} \partial_{t}^{j} f\right|$, when $[f]_{k+\alpha ; D}$ and $\langle f\rangle_{k+\alpha ; D}$ are finite. If $f$ is a function not depending on $t$, we keep the definition above by considering a function $\tilde{f}$ defined on $\Omega \times \mathbb{R}, \Omega \subset \mathbb{R}^{n}$, such that $\tilde{f}(x, t)=f(x)$ for every $(x, t) \in \Omega \times \mathbb{R}$, and we shall say that $f \in C^{k, \alpha}(\Omega)$ whenever $\tilde{f} \in C^{k, \alpha}(\Omega \times \mathbb{R})$. Throughout the paper we will make use of standard Sobolev spaces. We refer the reader to [18] for details.

Definition 2.1. Let $\Omega$ be a domain in $\mathbb{R}^{n}$. Given $\alpha, \alpha \in(0,1]$ and $k \in \mathbb{N}$, we say that $\partial \Omega$ is of class $C^{k, \alpha}$ with constants $r_{0}, L$ if for any $P \in \partial \Omega$ there exists a rigid transformation of $\mathbb{R}^{n}$ under which we have $P \equiv 0$ and

$$
\Omega \cap B_{r_{0}}=\left\{x \in B_{r_{0}}: x_{n}>\varphi\left(x^{\prime}\right)\right\},
$$

where $\varphi$ is a $C^{k, \alpha}$ function on $B_{r_{0}}^{\prime}$ satisfying the condition $\varphi(0)=\left|\nabla_{x^{\prime}} \varphi(0)\right|=0$ and $\|\varphi\|_{C^{k, \alpha}\left(B_{r_{0}}^{\prime}\right)} \leq L r_{0}$.

Remark 2.2. We have chosen to normalize all norms in such a way that their terms are dimensional homogeneous and coincide with the standard definition when $r_{0}=1$. For instance, for any $\varphi \in C^{k, \alpha}\left(B_{r_{0}}^{\prime} \times\left(-r_{0}^{2}, r_{0}^{2}\right)\right)$ we set

$$
\begin{gathered}
\|\varphi\|_{C^{k, \alpha}\left(B_{r_{0}}^{\prime} \times\left(-r_{0}^{2}, r_{0}^{2}\right)\right)}=\sum_{l=0}^{k} r_{0}^{l} \sum_{|\beta|+2 j=l}\left\|\partial_{x}^{\beta} \partial_{t}^{j} \varphi\right\|_{L^{\infty}\left(B_{r_{0}}^{\prime} \times\left(-r_{0}^{2}, r_{0}^{2}\right)\right)} \\
+r_{0}^{k+\alpha}\left(\langle\varphi\rangle_{k+\alpha ; B_{r_{0}}^{\prime} \times\left(-r_{0}^{2}, r_{0}^{2}\right)}+[\varphi]_{k+\alpha ; B_{r_{0}}^{\prime} \times\left(-r_{0}^{2}, r_{0}^{2}\right)}\right) .
\end{gathered}
$$

Similarly we set

$$
\|u\|_{L^{2}\left(B_{r_{0}}^{\prime} \times\left(-r_{0}^{2}, r_{0}^{2}\right)\right)}=r_{0}^{-\frac{n+1}{2}}\left(\int_{B_{r_{0}}^{\prime} \times\left(-r_{0}^{2}, r_{0}^{2}\right)} u^{2} d x d t\right)^{1 / 2} .
$$

We shall use the letters $C, C_{0}, C_{1}, \ldots$ to denote constants. The value of these constants may change from line to line and their dependance will be specified everywhere they appear. 
Assumptions on the domain. Let $r_{0}, M, L$ be given positive numbers. We assume that $\Omega$ is a bounded domain in $\mathbb{R}^{n}$ such that

$$
|\Omega| \leq M r_{0}^{n},
$$

where $|\Omega|$ denotes the Lebesgue measure,

$$
\partial \Omega=\bar{A} \cup \bar{I}
$$

where $A$ and $I$ are open subsets of $\partial \Omega, A \cap I=\emptyset$, and

$$
\partial \Omega \text { is of class } C^{1,1} \text { with constants } r_{0}, L \text {. }
$$

Also, we denote by $\Sigma$ an open portion of $A$ so that there exists a point $P_{0} \in \Sigma$ such that

$$
\partial \Omega \cap B_{r_{0}}\left(P_{0}\right) \subset \Sigma .
$$

Assumptions on the boundary data. Given positive constants $E, \Phi_{0}, \Phi_{1}$, on the accessible part $A$ of the boundary of $\Omega$, we shall prescribe two different heat fluxes $g$ and $\tilde{g}$ such that

$$
\begin{gathered}
g, \tilde{g} \in C^{0,1}(A \times[0, T]), \\
\operatorname{supp} g, \operatorname{supp} \tilde{g} \subset A^{r_{0}} \times[0, T],
\end{gathered}
$$

where $A^{r_{0}}=\left\{x \in A: \operatorname{dist}(x, I)>r_{0}\right\}$,

$$
\|g\|_{C^{0,1}(A \times[0, T])},\|\tilde{g}\|_{C^{0,1}(A \times[0, T])} \leq E,
$$

$$
\exists t_{1} \in(0, T) \text { such that } g(x, t)=\widetilde{g}(x, t), \quad \text { for } 0 \leq t \leq t_{1}, x \in A .
$$

These last three assumptions on $g, \tilde{g}$ can be understood as visibility conditions:

$$
\begin{gathered}
g \geq 0 \text { in } A^{r_{0}} \times[0, T], \\
g(x, t) \geq \Phi_{1} r_{0}^{-1}>0, \text { in } A^{2 r_{0}} \times[0, T],
\end{gathered}
$$

assuming $A^{2 r_{0}} \neq \emptyset$,

$$
\left\|\frac{\tilde{g}}{g}-\left(\frac{\tilde{g}}{g}\right)_{A^{2 r_{0} \times[0, T]}}\right\|_{L^{2}\left(A^{2 r_{0} \times[0, T]}\right)} \geq \Phi_{0}>0,
$$

where

$$
\left(\frac{\tilde{g}}{g}\right)_{A^{2 r_{0} \times[0, T]}}=\frac{1}{\mid A^{2 r_{0} \mid T}} \int_{A^{2 r_{0} \times[0, T]}} \frac{\tilde{g}}{g} d \sigma d t .
$$

Remark 2.3. We observe that, being $\left(\frac{\tilde{g}}{g}\right)_{A^{2 r_{0} \times[0, T]}}$ the argmin of the function

$$
\begin{aligned}
f: \quad \mathbb{R} & \rightarrow \mathbb{R} \\
c & \mapsto\left\|\frac{\tilde{g}}{g}-c\right\|_{L^{2}\left(A^{\left.2 r_{0} \times[0, T]\right)}\right.},
\end{aligned}
$$

we have that (2.2g) leads to

$$
\left\|\frac{\tilde{g}}{g}-c\right\|^{2} \geq \Phi_{0}, \quad \forall c \in \mathbb{R} .
$$

In this respect the hypothesis (2.2g) might be interpreted as a linear independence type condition in an integral form. 
Assumptions on the surface impedance. Given a positive number $\bar{\gamma}$, the surface impedance $\gamma$ of the unknown boundary $I$ is such that

$$
\gamma \in C^{0,1}(I \times[0, T]), \quad \text { with } \quad \operatorname{supp} \gamma \subset I \times[0, T]
$$

and

$$
0 \leq \gamma(x, t) \leq \bar{\gamma} r_{0}^{-1}
$$

Remark 2.4. By [17, Theorem 6.46, page 141, if (2.1a)-(2.1c), (2.2a), (2.2b), (2.3) are fulfilled, there exists a unique solution $u$ of the problem (1.1),$u \in C^{1, \alpha}(\bar{\Omega} \times$ $[0, T])$ such that

$$
\|u\|_{C^{1, \alpha}(\bar{\Omega} \times[0, T])} \leq C_{0}\|g\|_{C^{0,1}(A \times[0, T])}, \quad \forall \alpha \in(0,1),
$$

where $C_{0}$ is a positive constant depending $n, \Omega, \bar{\gamma}$.

From now on we shall fix an $\alpha, \alpha \in[1 / 2,1)$.

We denote by $\Omega_{i}, i=1,2$, two bounded domains in $\mathbb{R}^{n}$ satisfying (2.1) such that

$$
\partial \Omega_{i}=\bar{A} \cup \bar{I}_{i}, \quad A \cap I_{i}=\emptyset, \quad i=1,2,
$$

where the accessible part $A$ of the boundary is the same for both sets and by $\gamma_{i}(x, t)$, $i=1,2$, the boundary impedance on $I_{i}, i=1,2$, respectively satisfying (2.3).

In the sequel we shall refer to the numbers $M, L, r_{0}, \Phi_{0}, \Phi_{1}, \bar{\gamma}, r_{0}^{2} / T, r_{0}^{2} / t_{1}$, as the a priori data.

We denote then by $I_{i}^{r_{0}}=\left\{x \in I_{i}: \operatorname{dist}(x, A)>r_{0}\right\}$.

Theorem 2.5. For $i=1,2$, let $u_{i} \in C^{1, \alpha}(\bar{\Omega} \times[0, T])$ be the solution to (1.1) when $\Omega=\Omega_{i}, \gamma=\gamma_{i}$ and let $\tilde{u}_{i} \in C^{1, \alpha}(\bar{\Omega} \times[0, T])$ be the solution to (1.1) when $\Omega=\Omega_{i}$, $\gamma=\gamma_{i}, g=\tilde{g}$. Let (2.1a) (2.3b) be satisfied. If, given $\varepsilon>0$, we have

$$
\begin{aligned}
&\left\|u_{1}-u_{2}\right\|_{L^{2}\left(\Sigma \times\left(t_{1}, T\right)\right)} \leq \varepsilon, \\
&\left\|\tilde{u}_{1}-\tilde{u}_{2}\right\|_{L^{2}\left(\Sigma \times\left(t_{1}, T\right)\right)} \leq \varepsilon,
\end{aligned}
$$

then

$$
d_{\mathcal{H}}\left(\bar{\Omega}_{1}, \bar{\Omega}_{2}\right) \leq r_{0} \eta(\varepsilon),
$$

where $\eta$ is a continuous increasing function on $[0,+\infty)$ satisfying

$$
\eta(s) \leq C|\log s|^{-\beta},
$$

for every $0<s<1$, with $C>0$ and $\beta$ depending on the a priori data only. Furthermore, for any $t \in\left(t_{1}, T\right)$,

$$
\sup _{\substack{P \in I_{1} r_{0} \\ Q \in B_{2 \eta(\varepsilon)}(P) \cap I_{2}{ }^{r_{0}}}}\left|\gamma_{2}(Q, t)-\gamma_{1}(P, t)\right| \leq r_{0}^{-1} \eta(\varepsilon),
$$

where $\eta$ is defined as in (2.7) up to a possible replacement of constants $C$ and $\beta$.

Here $d_{\mathcal{H}}$ stands for the Hausdorff distance. 


\section{Proof of Theorem 2.5}

We first observe that the solution $u_{1} \in C^{1, \alpha}\left(\bar{\Omega}_{1} \times[0, T]\right)$ of problem (1.1) with boundary data $g$ satisfying (2.2e) is such that

$$
u_{1}(x, t)>0, \text { in } \Omega_{1} \times(0, T] .
$$

Namely, by contradiction, if

$$
\min _{\bar{\Omega}_{1} \times[0, T]} u_{1}(x, t)=u_{1}(\bar{x}, \bar{t}) \leq 0,
$$

then, by maximum principle, the point $(\bar{x}, \bar{t})$ belongs to the parabolic boundary.

If $(\bar{x}, \bar{t}) \in A \times(0, T]$, by the Hopf lemma we would have $\frac{\partial u_{1}}{\partial \nu}(\bar{x}, \bar{t})<0$, which contradicts (2.2e). If $(\bar{x}, \bar{t}) \in I \times(0, T]$, again by the Hopf lemma we would have $\frac{\partial u_{1}}{\partial \nu}(\bar{x}, \bar{t})<0$, which contradicts the Robin condition that $u_{1}$ satisfies $I$ because

$$
\frac{\partial u_{1}}{\partial \nu}(\bar{x}, \bar{t})=-\gamma u_{1}(\bar{x}, \bar{t}) \geq 0
$$

Then $(\bar{x}, \bar{t})=(\bar{x}, 0)$,

$$
\min _{\bar{\Omega}_{1} \times[0, T]} u_{1}(x, t)=u_{1}(\bar{x}, 0)=0
$$

and we get (3.1).

The same is true for $u_{2}(x, t)$.

By (3.1) we can define, for $i=1,2$,

$$
\lambda_{i}(x, t)=\frac{\widetilde{u}_{i}(x, t)}{u_{i}(x, t)}-1, \text { in } \bar{\Omega}_{i} \times\left[t_{1}, T\right] .
$$

By straightforward calculation we notice that $\lambda_{i}(x, t)$ satisfies the problem

$$
\begin{cases}\partial_{t} \lambda_{i}(x, t)=\operatorname{div}\left(u_{i}^{2} \nabla \lambda_{i}(x, t)\right), & \text { in } \Omega_{i} \times\left[t_{1}, T\right], \\ \lambda_{i}\left(x, t_{1}\right)=0, & \text { in } \Omega_{i}, \\ u_{i}^{2} \frac{\partial \lambda_{i}}{\partial \nu}(x, t)=u_{i} \tilde{g}(x, t)-\tilde{u}_{i} g(x, t), & \text { on } A \times\left[t_{1}, T\right], \\ u_{i}^{2} \frac{\partial \lambda_{i}}{\partial \nu}(x, t)=0, & \text { on } I_{i} \times\left[t_{1}, T\right] .\end{cases}
$$

With the change of variable (3.2) we can deal with the new problem (3.3), where we have a homogeneous Neumann condition on $I_{i} \times\left[t_{1}, T\right]$.

By standard estimates of solutions of parabolic problems in [17], by (2.2b), (2.2c), (2.4), we have

$$
\left\|\lambda_{i}\right\|_{C^{1, \alpha}\left(\bar{\Omega}_{i} \times\left[t_{1}, T\right]\right)} \leq C,
$$

where $\alpha \in(0,1)$ and $C$ depends on the a priori data only.

In the next propositions, whose proofs are postponed to Section 4, we provide stability estimates of unique continuation from Cauchy data when (2.5) holds true, and then a lower bound on $u$, where $u$ is a solution to (1.1), and a lower bound of the integral of $\lambda_{i}$ in terms of the boundary data.

The proof of Theorem 2.5 will be obtained from such a sequence of propositions.

We shall denote by $G$ the connected component of $\Omega_{1} \cap \Omega_{2}$ such that $A \subset \bar{G}$. 
Proposition 3.1 (Stability estimates of unique continuation from Cauchy data). Let the hypothesis of Theorem 2.5 be satisfied. Then there exists a positive constant $C$ depending on the a priori data only such that for $i=1,2$

$$
\int_{\Omega_{i} \backslash G} u_{i}^{2}(x, t) \lambda_{i}^{2}(x, t) d x \leq C r_{0}^{n} \eta(\varepsilon) \quad \forall t \in\left[t_{1}, T\right],
$$

where $\eta$ is an increasing continuous function on $[0,+\infty)$ which satisfies

$$
\eta(s) \leq(\log |\log s|)^{-\beta_{1}},
$$

for every $0<s<1$, with $\beta_{1}>0$.

Proposition 3.2 (Improved stability estimates). Let the hypothesis of Proposition 3.1 be fulfilled. In addition, assume there exist constants $L_{1}>0$ and $r_{1}, 0<r_{1} \leq r_{0}$, such that $\partial G$ is of Lipschitz class with constants $r_{1}$ and $L_{1}$. Then there exists a positive constant $C$ depending on the a priori data and on $L_{1}, \frac{r_{0}}{r_{1}}$ such that, for $i=1,2$, (3.5) holds, where $\eta$ is an increasing continuous function on $[0,+\infty)$ satisfying

$$
\eta(s) \leq|\log s|^{-\beta_{2}},
$$

for every $0<s<1$, with $\beta_{2}>0$.

Proposition 3.3 (Lower bound on $u$ ). Let $u \in C^{1, \alpha}(\bar{\Omega} \times[0, T])$ be a solution to (1.1), where (2.1), (2.2a)-(2.2c), (2.2e), (2.2f), (2.3) are satisfied. Let $t_{1} \in(0, T)$. Then there exists a positive constant $c_{0}, 0<c_{0}<1$, depending on the a priori data except $\Phi_{0}$, such that

$$
u(x, t) \geq c_{0} \quad \text { for } t \geq t_{1}, x \in \Omega .
$$

Proposition 3.4 (Lower bound on $\lambda_{i}$ ). Let the assumptions of Theorem 2.5 be satisfied. For every $\rho>0$ and for every $x_{0} \in \Omega_{\rho}$, we have for $i=1,2$,

$$
\int_{t_{1}}^{T} \int_{B_{\rho}\left(x_{0}\right)} \lambda_{i}^{2}(x, t) d x d t \geq C_{\rho} r_{0}^{n+2} \Phi_{0},
$$

where $C_{\rho}>0$ is a constant depending on the a priori data and $\rho$ only.

To better deal with the Hausdorff distance, we recall the modified distance introduced in [2] (see also [10]).

Definition 3.5. We call the modified distance between $\Omega_{1}$ and $\Omega_{2}$ the number

$$
d_{m}\left(\Omega_{1}, \Omega_{2}\right)=\max \left\{\sup _{x \in \partial \Omega_{1}} \operatorname{dist}\left(x, \bar{\Omega}_{2}\right), \sup _{x \in \partial \Omega_{2}} \operatorname{dist}\left(x, \bar{\Omega}_{1}\right)\right\} .
$$

Note that

$$
d_{m}\left(\Omega_{1}, \Omega_{2}\right) \leq d_{\mathcal{H}}\left(\bar{\Omega}_{1}, \bar{\Omega}_{2}\right),
$$

but, in general, the reverse inequality does not hold. However, the following result holds true.

Proposition 3.6 ([2, Proposition 3.6). Let $\Omega_{1}$ and $\Omega_{2}$ be bounded domains satisfying (2.1c). There exist numbers $d_{0}>0, \tilde{r} \in\left(0, r_{0}\right]$, such that $\frac{d_{0}}{r_{0}}$ and $\frac{\tilde{r}}{r_{0}}$ depend on $L$ only and the following facts hold true. If

$$
d_{\mathcal{H}}\left(\bar{\Omega}_{1}, \bar{\Omega}_{2}\right) \leq d_{0},
$$


then there exists an absolute constant $C>0$ such that

$$
d_{\mathcal{H}}\left(\bar{\Omega}_{1}, \bar{\Omega}_{2}\right) \leq C d_{m}\left(\Omega_{1}, \Omega_{2}\right),
$$

and any connected component of $\Omega_{1} \cap \Omega_{2}$ has a boundary of Lipschitz class with constants $\tilde{r}, L_{1}$ where $\tilde{r}$ is as above and $L_{1}>0$ depends on $L$ only.

The last tool we need for the proof of Theorem 2.5 is related to the quantitative form of the unique continuation property.

Theorem 3.7 (Two-sphere one-cylinder inequality at the boundary). Let $\lambda, \Lambda$ and $R$ be positive numbers, with $\lambda \in(0,1]$ and $t_{0} \in \mathbb{R}$. Let $\mathcal{L}$ be a parabolic operator $\mathcal{L}=\partial_{i}\left(g^{i j}(x, t) \partial_{j}\right)-\partial_{t}$, where $\left\{g^{i j}(x, t)\right\}_{i, j=1}^{n}$ is a real symmetric $n \times n$ matrix. For $\xi \in \mathbb{R}^{n},(x, t),(y, \tau) \in \mathbb{R}^{n+1}$, assume that

$$
\lambda|\xi|^{2} \leq \sum_{i, j=1}^{n} g^{i j}(x, t) \xi_{i} \xi_{j} \leq \lambda^{-1}|\xi|^{2}
$$

and

$$
\left(\sum_{i, j=1}^{n}\left(g^{i j}(x, t)-g^{i j}(y, \tau)\right)^{2}\right)^{1 / 2} \leq \frac{\Lambda}{R}\left(|x-y|^{2}+|t-\tau|\right)^{1 / 2} .
$$

Let $u \in H^{2,1}\left(Q_{R, \varphi}^{t_{0}}\right)$ be such that

$$
|\mathcal{L} u| \leq \Lambda\left(\frac{|\nabla u|}{R}+\frac{|u|}{R^{2}}\right), \quad \text { in } Q_{R, \varphi}^{t_{0}}
$$

and

$$
g^{i j} \frac{\partial u}{\partial x_{j}}(x, t) \nu_{i}=0, \quad \forall(x, t) \in \Gamma_{R, \varphi}^{t_{0}} .
$$

There exist constants $s_{1} \in(0,1)$ and $C, C>0$, depending on $\lambda, \Lambda$ and $L$ only such that for every $r, \rho, 0<r \leq \rho \leq s_{1} R$ we have

$$
\int_{B_{\rho} \cap \Omega} u^{2}\left(x, t_{0}\right) d x \leq \frac{C R^{2}}{\rho^{2}}\left(R^{-2} \int_{Q_{R, \varphi}^{t_{0}}} u^{2} d x d t\right)^{1-\theta}\left(\int_{B_{r} \cap \Omega} u^{2}\left(x, t_{0}\right) d x\right)^{\theta}
$$

where $\theta=\frac{1}{C \log \frac{R}{r}}$.

Proof. The proof can be obtained along the lines of [24, Theorem 3.3.5] through slight modifications due to the different boundary condition we have on $\Gamma_{R, \varphi}^{t_{0}}$ (see also [11], where a similar problem is studied).

An inequality similar to (3.12) can be obtained for cylinder and spheres entirely contained in the domain $\Omega$. We refer the interested reader to [24, Theorem 3.3.3].

We are now ready to begin with the proof of Theorem 2.5.

Proof of Theorem 2.5. For the sake of brevity we denote $d=d_{\mathcal{H}}\left(\bar{\Omega}_{1}, \bar{\Omega}_{2}\right)$ and $d_{m}=$ $d_{m}\left(\bar{\Omega}_{1}, \bar{\Omega}_{2}\right)$. The proof follows the lines of the proof of [8, Theorem 4.1]. We shall sketch only the main items, using Propositions 3.1, 3.2, 3.3, 3.4, 3.6 and Theorem 3.7 .

Let us prove first that if $\eta>0$ is such that

$$
\int_{t_{1}}^{T} \int_{\Omega_{1} \backslash G} u_{1}^{2}(x, t) \lambda_{1}^{2}(x, t) d x d t \leq r_{0}^{n+2} \eta,
$$


then there exists a constant $C$, depending on the a priori data only, such that

$$
d_{m} \leq C r_{0} \eta^{K}
$$

where $K$ depends on the a priori data only.

By Proposition 3.3 (the lower bound on $u$ ) and by (3.13), we also have

$$
\int_{t_{1}}^{T} \int_{\Omega_{1} \backslash G} \lambda_{1}^{2}(x, t) d x d t \leq c_{0}^{-2} r_{0}^{n+2} \eta .
$$

We may assume, without loss of generality, that there exists $x_{0}=0 \in I_{1} \subseteq \partial \Omega_{1}$ such that

$$
\operatorname{dist}\left(x_{0}, \bar{\Omega}_{2}\right)=d_{m}
$$

which implies, by (3.15),

$$
\int_{t_{1}}^{T} \int_{B_{d_{m}} \cap \Omega_{1}} \lambda_{1}^{2}(x, t) d x d t \leq c_{0}^{-2} r_{0}^{n+2} \eta
$$

We now apply to $\lambda_{1}(x, t)$ the two-sphere one-cylinder inequality at the boundary, that is, Theorem 3.7, with $r=d_{m}, \rho=s_{1} r_{0}$ and $R=r_{0}$. First we observe that, by Proposition 3.3 and by the definition of $\lambda_{1}$,

$$
\begin{aligned}
& \left(\int_{Q_{r_{0}, \varphi}^{t_{0}}} \lambda_{1}^{2}(x, t) d x d t\right) \leq c_{0}^{-2} \int_{Q_{r_{0}, \varphi}^{t_{0}}}\left|\widetilde{u}_{1}(x, t)-u_{1}(x, t)\right|^{2} d x d t \\
\leq & c_{0}^{-2} \int_{0}^{T} \int_{\Omega_{1}}\left|\widetilde{u}_{1}(x, t)-u_{1}(x, t)\right|^{2} d x d t=c_{0}^{-2} A r_{0}^{n+2}, \quad \forall t_{0} \in\left[t_{1}, T\right],
\end{aligned}
$$

where $A=r_{0}^{-(n+2)} \int_{0}^{T} \int_{\Omega_{1}}\left|\widetilde{u}_{1}(x, t)-u_{1}(x, t)\right|^{2} d x d t$.

Integrating in (3.12) over the interval $\left[t_{1}, T\right]$, applying Holder's inequality with exponent $\frac{1}{\theta}$, we get, assuming $d_{m} \leq s_{1} r_{0}$ (since the other case follows trivially),

$$
\int_{t_{1}}^{T} \int_{B_{\rho} \cap \Omega_{1}} \lambda_{1}^{2}(x, t) d x d t \leq \frac{C}{s_{1}^{2}}\left(\frac{1}{c_{0}^{2}} A r_{0}^{n+2}\right)^{1-\theta} \times\left(\int_{t_{1}}^{T} \int_{B_{d_{m}} \cap \Omega_{1}} \lambda_{1}^{2}(x, t) d x d t\right)^{\theta}
$$

with $\theta=\frac{1}{C \log \left(\frac{r_{0}}{d_{m}}\right)}$.

Let $x_{1} \in B_{s_{1} r_{0}} \cap \Omega_{1}$ such that $x_{1}=0-\bar{r} \sqrt{1+L^{2}} \nu$. Then $B_{\bar{r}}\left(x_{1}\right)$, with $\bar{r}=$ $\frac{d_{m}}{2\left(1+\sqrt{1+L^{2}}\right)}$, is compactly contained in $B_{s_{1} r_{0}} \cap \Omega_{1}$ and, by consequence,

$$
\int_{t_{1}}^{T} \int_{B_{\bar{r}}\left(x_{1}\right)} \lambda_{1}^{2}(x, t) d x d t \leq \int_{t_{1}}^{T} \int_{B_{\rho} \cap \Omega_{1}} \lambda_{1}^{2}(x, t) d x d t .
$$

By Proposition 3.4 (the lower bound on $\lambda_{1}$ ), we get

$$
C_{1} r_{0}^{n+2} \Phi_{0} \leq \int_{t_{1}}^{T} \int_{B_{\bar{r}}\left(x_{1}\right)} \lambda_{1}^{2}(x, t) d x d t
$$

where $C_{1}$ depends on the a priori data.

Finally, collecting (3.16), (3.18), (3.19), (3.20), we get

$$
C_{1} \Phi_{0} \leq \frac{C}{s_{1}^{2}}\left(\frac{1}{c_{0}^{2}} A\right)^{1-\theta} c_{0}^{-2 \theta} \eta^{\theta},
$$


that is,

$$
\Phi_{0} \leq C_{4}\left(\frac{\eta}{A}\right)^{\frac{1}{C \log \left(\frac{r_{0}}{d_{m}}\right)}}
$$

where $C_{4}$ depends on the a priori data.

Assuming $\frac{\eta}{A}<1$ (on the contrary (3.14) is trivial) and developing (3.21), we conclude that

$$
\left(\frac{d_{m}}{r_{0}}\right)^{C} \leq\left(\frac{\eta}{A}\right)^{\frac{1}{\left|\frac{\Phi_{0}}{\log _{4}}\right|}}
$$

that is,

$$
d_{m} \leq C_{5} r_{0} \eta^{\frac{1}{\left|C \log \frac{\Phi_{0}}{C_{4}}\right|}}=C_{5} r_{0} \eta^{K}
$$

where $C_{5}, K$ depend on the a priori data.

Let us now consider the Hausdorff distance $d$. With no loss of generality, we may assume that there exists $y_{0} \in \bar{\Omega}_{1}$ such that $\operatorname{dist}\left(y_{0}, \bar{\Omega}_{2}\right)=d$. Denote this by $h=\operatorname{dist}\left(y_{0}, \partial \Omega_{1}\right)$. We distinguish three cases:

i) $h \leq \frac{d}{2}$.

Taking $z_{0} \in \partial \Omega_{1}$ such that $\left|y_{0}-z_{0}\right|=h$, we have that $d_{m} \geq \operatorname{dist}\left(z_{0}, \bar{\Omega}_{2}\right) \geq$ $d-h \geq \frac{d}{2}$, so that $d \leq 2 d_{m}$ and we get (3.14) for $d$, instead of $d_{m}$.

ii) $h>\max \left\{\frac{d}{2}, \frac{d_{0}}{2}\right\}$, with $d_{0}$ introduced in Proposition 3.6 .

We want to apply to $\lambda_{1}$ the two-sphere one-cylinder inequality in the interior that is [24, Theorem 3.3.3], maintaining the notation of Theorem 3.7 Let us denote this by $d_{1}=\min \left\{\frac{d}{2}, \frac{s_{1} d_{0}}{4}\right\}$. Since $d_{1}<d$, we have $B_{d_{1}}\left(y_{0}\right) \subset \Omega_{1} \backslash \Omega_{2}$; since $h>d_{0} / 2>s_{1} d_{0} / 2$, we have $B_{s_{2} d_{0} / 2}\left(y_{0}\right) \subset \Omega_{1}$. We apply the above cited theorem with $r=d_{1}, \rho=s_{1} d_{0} / 2, R=d_{0}$. Proceeding as in (3.17) (3.21) we get (3.14) for d.

iii) $\frac{d}{2}<h \leq \frac{d_{0}}{2}$.

In this case $d<d_{0}$, and by Proposition 3.5 we get (3.14) for $d$.

Finally, by using Propositions 3.1, 3.2, we get the thesis (2.8).

Let us now prove (2.8).

First we observe that, in general, the Hausdorff distances $d_{\mathcal{H}}\left(\bar{\Omega}_{1}, \bar{\Omega}_{2}\right)$ and $d_{\mathcal{H}}\left(\partial \Omega_{1}, \partial \Omega_{2}\right)$ are not equivalent. However, in our regularity assumptions, the estimate

$$
d_{\mathcal{H}}\left(\partial \Omega_{1}, \partial \Omega_{2}\right) \leq r_{0} \eta(\varepsilon)
$$

can be derived from (2.6) using the arguments contained in the proof of Proposition 3.6 in [2]. We consider a point $P \in I_{1}{ }^{r_{0}}$, a point $Q \in B_{2 \eta(\varepsilon)}(P) \cap I_{2}{ }^{r_{0}}$ and $t \in\left(t_{1}, T\right)$. With no loss of generality we may assume that $P, Q \in \bar{\Omega}_{1}$. Hence we have that for any $t \in\left(t_{1}, T\right)$,

$$
\begin{aligned}
\left|\gamma_{2}(Q, t)-\gamma_{1}(P, t)\right| \leq & \left|\frac{\partial u_{1}}{\partial \nu}(P, t) \frac{1}{u_{1}(P, t)}-\frac{\partial u_{1}}{\partial \nu}(Q, t) \frac{1}{u_{1}(Q, t)}\right| \\
& +\left|\frac{\partial u_{2}}{\partial \nu}(Q, t) \frac{1}{u_{2}(Q, t)}-\frac{\partial u_{1}}{\partial \nu}(Q, t) \frac{1}{u_{1}(Q, t)}\right|
\end{aligned}
$$


We can split the first term on the right hand side of (3.23) as follows:

$$
\begin{aligned}
\mid \frac{\partial u_{1}}{\partial \nu} & (P, t) \frac{1}{u_{1}(P, t)}-\frac{\partial u_{1}}{\partial \nu}(Q, t) \frac{1}{u_{1}(Q, t)} \mid \\
& \leq\left|\frac{\partial u_{1}}{\partial \nu}(P, t) \frac{1}{u_{1}(P, t)}-\frac{\partial u_{1}}{\partial \nu}(Q, t) \frac{1}{u_{1}(P, t)}\right| \\
& +\left|\frac{\partial u_{1}}{\partial \nu}(Q, t) \frac{1}{u_{1}(P, t)}-\frac{\partial u_{1}}{\partial \nu}(Q, t) \frac{1}{u_{1}(Q, t)}\right| .
\end{aligned}
$$

From Remark 2.4 and Proposition 3.3 we can infer that

$$
\left|\frac{\partial u_{1}}{\partial \nu}(P, t) \frac{1}{u_{1}(P, t)}-\frac{\partial u_{1}}{\partial \nu}(Q, t) \frac{1}{u_{1}(Q, t)}\right| \leq C|P-Q| r_{0}^{-2},
$$

where $C>0$ is a constant depending on the a priori data only. Hence by (3.22) we can infer that

$$
\left|\frac{\partial u_{1}}{\partial \nu}(P, t) \frac{1}{u_{1}(P, t)}-\frac{\partial u_{1}}{\partial \nu}(Q, t) \frac{1}{u_{1}(Q, t)}\right| \leq r_{0}^{-1} \eta(\varepsilon),
$$

up to a possible replacement of the constants $C$ and $\beta$ in (2.7).

Analogously we can split the second term on the right hand side of (3.23) as follows:

$$
\begin{aligned}
& \left|\frac{\partial u_{2}}{\partial \nu}(Q, t) \frac{1}{u_{2}(Q, t)}-\frac{\partial u_{1}}{\partial \nu}(Q, t) \frac{1}{u_{1}(Q, t)}\right| \\
& \quad \leq\left|\frac{\partial u_{2}}{\partial \nu}(Q, t) \frac{1}{u_{2}(Q, t)}-\frac{\partial u_{1}}{\partial \nu}(Q, t) \frac{1}{u_{2}(Q, t)}\right| \\
& \quad+\left|\frac{\partial u_{1}}{\partial \nu}(Q, t) \frac{1}{u_{2}(Q, t)}-\frac{\partial u_{1}}{\partial \nu}(Q, t) \frac{1}{u_{1}(Q, t)}\right| .
\end{aligned}
$$

From Remark 2.4 and Proposition 3.3 we can infer that

$$
\begin{aligned}
& \left|\frac{\partial u_{2}}{\partial \nu}(Q, t) \frac{1}{u_{2}(Q, t)}-\frac{\partial u_{1}}{\partial \nu}(Q, t) \frac{1}{u_{1}(Q, t)}\right| \\
\leq & C\left|\frac{\partial u_{2}}{\partial \nu}(Q, t)-\frac{\partial u_{1}}{\partial \nu}(Q, t)\right|+C\left|u_{1}(Q, t)-u_{2}(Q, t)\right| r_{0}^{-1},
\end{aligned}
$$

where $C>0$ is a constant depending on the a priori data only. Adapting to the present case the techniques of the proof of Proposition [3.2, we have that for any $t \in\left(t_{1}, T\right)$,

$$
\left\|u_{1}(\cdot, t)-u_{2}(\cdot, t)\right\|_{C^{1}\left(I_{2} r_{0}\right)} \leq \eta(\varepsilon) .
$$

Hence we have that

$$
\left|\frac{\partial u_{2}}{\partial \nu}(Q, t) \frac{1}{u_{2}(Q, t)}-\frac{\partial u_{1}}{\partial \nu}(Q, t) \frac{1}{u_{1}(Q, t)}\right| \leq r_{0}^{-1} \eta(\varepsilon) .
$$

Combining (3.25) and (3.26) we obtain that for any $t \in\left(t_{1}, T\right)$,

$$
\left|\gamma_{2}(Q, t)-\gamma_{1}(P, t)\right| \leq r_{0}^{-1} \eta(\varepsilon) .
$$

Being such an estimate independent from $P, Q$ and $t$, the thesis (2.8) follows. 


\section{Proofs of Propositions 3.1, 3.2, 3.3, 3.4}

We proceed with the proof of Proposition 3.1. For this purpose we recall a result in [8] that will be used in the following (see also [10,23]).

Theorem 4.1. Let $\Omega$ be a domain satisfying (2.1c). Let $\Sigma$ be an open portion of $\partial \Omega$ satisfying

$$
\partial \Omega \cap B_{r_{0}}\left(P_{1}\right) \subset \Sigma,
$$

for some $P_{1} \in \Sigma$. Let $u \in H^{2,1}(\Omega \times(0, T))$ be a solution to

$$
\left\{\begin{array}{l}
u_{t}=\Delta u, \quad \text { in } \Omega \times(0, T) \\
u(x, 0)=0, \quad x \in \Omega
\end{array}\right.
$$

satisfying

$$
\|u\|_{H^{3 / 2,3 / 4}(\Sigma \times(0, T))} \leq C \bar{\varepsilon}, \quad\left\|\frac{\partial u}{\partial \nu}\right\|_{H^{1 / 2,1 / 4}(\Sigma \times(0, T))} \leq C \bar{\varepsilon},
$$

where $C$ depends on $T, r_{0}$. Then, for every $t_{0} \in[0, T]$, we have

$$
\left\|u\left(\cdot, t_{0}\right)\right\|_{L^{2}\left(B_{\bar{\theta} r_{0}}\left(P_{2}\right)\right)} \leq C\|u\|_{H^{2,1}(\Omega \times(0, T))}^{1-\bar{\tau}} \bar{\varepsilon}^{\bar{\tau}},
$$

where $P_{2}=P_{1}-\bar{\theta} r_{0} \nu, \nu$ is the outer unit normal to $\Omega$ at $P_{1}, \bar{\tau}, 0<\bar{\tau}<1$, is an absolute constant, $\bar{\theta}, 0<\bar{\theta}<1 / 2$, depends on $L$ only, and $C \geq 1$ depends on $L$ and $r_{0}^{2} / T$ only.

Proof of Proposition 3.1. We prove the proposition for $i=1$, the other case $i=2$ being analogous. In [16] it is proved that there exists a function $\widetilde{d}(x)$, labeled regularized distance, $\widetilde{d} \in C^{2}\left(\Omega_{1}\right) \cap C^{1,1}\left(\bar{\Omega}_{1}\right)$, such that the following facts hold:

$$
\begin{aligned}
& \text { i) } \xi_{1} \leq \frac{\operatorname{dist}\left(x, \partial \Omega_{1}\right)}{\widetilde{d}(x)} \leq \xi_{2}, \\
& \text { ii) }|\nabla \widetilde{d}(x)| \geq c_{1} \text { for every } x \text { such that } \operatorname{dist}\left(x, \partial \Omega_{1}\right) \leq b r_{0}, \\
& \text { iii) }\|\widetilde{d}\|_{C^{1,1}} \leq c_{2} r_{0},
\end{aligned}
$$

where $\xi_{1}, \xi_{2}, c_{1}, c_{2}, b$ are positive constants depending on $L$ only (see also [2, Lemma $5.2]$ ). For $r>0$ we define

$$
\widetilde{\Omega}_{1, r}=\left\{x \in \Omega_{1}: \widetilde{d}(x)>r\right\} .
$$

By [2, Lemma 5.3], there exists a constant $a$, depending on $L$ only, such that for every $r, 0<r \leq a r_{0}, \widetilde{\Omega}_{1, r}$ is connected with boundary of class $C^{1}$ and the following facts hold true:

$$
\begin{aligned}
& \xi_{1} r \leq \operatorname{dist}\left(x, \partial \Omega_{1}\right) \leq \xi_{2} r, \quad \forall x \in \partial \widetilde{\Omega}_{1, r}, \\
& \left|\Omega_{1} \backslash \widetilde{\Omega}_{1, r}\right| \leq \xi_{3} M r_{0}^{n-1} r \\
& \left|\partial \widetilde{\Omega}_{1, r}\right|_{n-1} \leq \xi_{4} M r_{0}^{n-1} .
\end{aligned}
$$

Also, for every $x \in \partial \tilde{\Omega}_{1, r}$, there exists $y \in \partial \Omega_{1}$ such that

$$
|y-x|=\operatorname{dist}\left(x, \partial \Omega_{1}\right), \quad|\nu(x)-\nu(y)| \leq \xi_{5} \frac{r}{r_{0}}
$$


where $\nu(x), \nu(y)$ denote the outer unit normal to $\widetilde{\Omega}_{1, r}$ at $x$ and to $\Omega_{1}$ at $y$ respectively. Here $\xi_{j}, j=1, \ldots, 5$, are constants depending on $L$ only.

Let us define $\theta=\min \left\{a, \frac{1}{16\left(1+L^{2}\right) \xi_{2}}\right\}$; since $\xi_{2} r_{0} \theta \leq \frac{r_{0}}{16}$, we have $\Sigma_{\xi_{2} r_{0} \theta}=\{x \in$ $\left.\Omega_{1}: \operatorname{dist}(x, \Sigma)=\xi_{2} r_{0} \theta\right\} \equiv\left\{x \in \Omega_{2}: \operatorname{dist}(x, \Sigma)=\xi_{2} r_{0} \theta\right\}$.

Let $\tilde{V}_{r}$ be the connected component of $\tilde{\Omega}_{1, r} \cap \tilde{\Omega}_{2, r}$ whose closure contains $\Sigma_{\xi_{2} r_{0} \theta}$. We have

$$
\begin{aligned}
& \Omega_{1} \backslash G \subset\left[\left(\Omega_{1} \backslash \tilde{\Omega}_{1, r}\right) \backslash G\right] \cup\left[\tilde{\Omega}_{1, r} \backslash \tilde{V}_{r}\right], \\
& \partial\left(\tilde{\Omega}_{1, r} \backslash \tilde{V}_{r}\right)=\tilde{\Gamma}_{1, r} \cup \tilde{\Gamma}_{2, r},
\end{aligned}
$$

where $\tilde{\Gamma}_{1, r}$ is the part of the boundary contained in $\partial \tilde{\Omega}_{1, r}$ and $\tilde{\Gamma}_{2, r}$ is the part contained in $\partial \tilde{\Omega}_{2, r} \cap \partial \tilde{V}_{r}$. For $t_{1} \leq t \leq T$, by (4.6)

$$
\begin{aligned}
& \int_{\Omega_{1} \backslash G} u_{1}^{2}(x, t) \lambda_{1}^{2}(x, t) d x \\
\leq & \int_{\left(\Omega_{1} \backslash \tilde{\Omega}_{1, r}\right) \backslash G} u_{1}^{2}(x, t) \lambda_{1}^{2}(x, t) d x+\int_{\tilde{\Omega}_{1, r} \backslash \tilde{V}_{r}} u_{1}^{2}(x, t) \lambda_{1}^{2}(x, t) d x .
\end{aligned}
$$

Since $u_{1}^{2}(x, t) \lambda_{1}^{2}(x, t)=\left(\tilde{u}_{1}(x, t)-u_{1}(x, t)\right)^{2}$, by (2.4), (2.2c), (4.2), there exists a constant $C$ depending on the a priori data, except $\Phi_{0}, \Phi_{1}$, such that, for $t_{1} \leq t \leq T$,

$$
\int_{\left(\Omega_{1} \backslash \tilde{\Omega}_{1, r}\right) \backslash G} u_{1}^{2}(x, t) \lambda_{1}^{2}(x, t) d x \leq C r r_{0}^{n-1} .
$$

Let us evaluate $\int_{\tilde{\Omega}_{1, r} \backslash \tilde{V}_{r}} u_{1}^{2}(x, t) \lambda_{1}^{2}(x, t) d x$. Recalling that $\lambda_{1}$ solves (3.3) and denoting by $\omega_{r}=\tilde{\Omega}_{1, r} \backslash \tilde{V}_{r}$, we get

$$
\int_{t_{1}}^{t} d s \int_{\omega_{r}} u_{1}^{2} \lambda_{1 t} \lambda_{1} d x=\int_{t_{1}}^{t} d s \int_{\partial \omega_{r}} u_{1}^{2} \frac{\partial \lambda_{1}}{\partial \nu} \lambda_{1} d \sigma-\int_{t_{1}}^{t} d s \int_{\omega_{r}} u_{1}^{2}\left|\nabla \lambda_{1}\right|^{2} d x,
$$

where $\nu$ is the outer normal to $\omega_{r}$.

We have, integrating by parts, the left hand side, and since $\lambda_{1}\left(x, t_{1}\right)=0$,

$$
\begin{aligned}
& \frac{1}{2} \int_{t_{1}}^{t} d s \int_{\omega_{r}} u_{1}^{2} \lambda_{1 t} \lambda_{1} d x=\left.\frac{1}{2} \int_{\omega_{r}} u_{1}^{2} \lambda_{1}^{2}\right|_{t_{1}} ^{t} d x-\int_{t_{1}}^{t} d s \int_{\omega_{r}} \lambda_{1}^{2} u_{1} u_{1 t} d x \\
= & \frac{1}{2} \int_{\omega_{r}} u_{1}^{2}(x, t) \lambda_{1}^{2}(x, t) d x-\int_{t_{1}}^{t} d s \int_{\omega_{r}} \lambda_{1}^{2} u_{1} u_{1 t} d x .
\end{aligned}
$$

Therefore plugging (4.9) into (4.10), we have

$$
\begin{aligned}
& \frac{1}{2} \int_{\omega_{r}} u_{1}^{2}(x, t) \lambda_{1}^{2}(x, t) d x+\int_{t_{1}}^{t} d s \int_{\omega_{r}} u_{1}^{2}\left|\nabla \lambda_{1}\right|^{2} d x \\
& =\int_{t_{1}}^{t} d s \int_{\omega_{r}} \lambda_{1}^{2} u_{1} u_{1 t} d x+\int_{t_{1}}^{t} d s \int_{\partial \omega_{r}} u_{1}^{2} \frac{\partial \lambda_{1}}{\partial \nu} \lambda_{1} d \sigma .
\end{aligned}
$$

By Proposition 3.3, (2.2c), (2.4), (3.4), we get

$$
\begin{aligned}
C_{1} \int_{\omega_{r}} \lambda_{1}^{2}(x, t) d x \leq \int_{t_{1}}^{t} d s \int_{\omega_{r}} \lambda_{1}^{2} u_{1} u_{1 t} d x+\int_{t_{1}}^{t} d s \int_{\partial \omega_{r}} u_{1}^{2} \frac{\partial \lambda_{1}}{\partial \nu} \lambda_{1} d \sigma \\
(4.11) \leq C_{2}\left(r_{0}^{-2} \int_{t_{1}}^{t} d s \int_{\omega_{r}} \lambda_{1}^{2} d x+T \max _{t_{1} \leq \theta \leq t} \int_{\partial \omega_{r}}\left|\frac{\partial \lambda_{1}(x, \theta)}{\partial \nu}\right| d \sigma\right),
\end{aligned}
$$


for $t_{1} \leq t \leq T$, where $C_{1}, C_{2}$ depend on a priori data, except $\Phi_{0}$. Denoting by $\bar{t}$, $\bar{t} \in\left[t_{1}, t\right]$, where the maximum is achieved, by the Gronwall inequality we obtain

$$
\int_{\omega_{r}} \lambda_{1}^{2}(x, t) d x \leq C T \int_{\partial \omega_{r}}\left|\frac{\partial \lambda_{1}(x, \bar{t})}{\partial \nu}\right| d \sigma, \quad \forall t \in\left[t_{1}, T\right],
$$

where $C$ is a constant depending on the a priori data, except $\Phi_{0}$.

Recalling that $\partial \omega_{r} \subset \tilde{\Gamma}_{1, r} \cup \tilde{\Gamma}_{2, r}$, we distinguish two situations: either $x \in \tilde{\Gamma}_{1, r}$ or $x \in \tilde{\Gamma}_{2, r}$. Let $x \in \tilde{\Gamma}_{1, r}$. By (4.1) dist $\left(x, \partial \Omega_{1}\right) \leq \xi_{2} r$. On the other hand $\operatorname{dist}(x, \Sigma)>\xi_{2} r$ (see [2, Proposition 3.1]). Hence there exists $y \in \partial \Omega_{1} \backslash \Sigma$ such that $|y-x|=\operatorname{dist}\left(x, \partial \Omega_{1}\right) \leq \xi_{2} r$. Since $\frac{\partial \lambda_{1}}{\partial \nu}=0$ on $I_{1} \times\left[t_{1}, T\right]$, by (3.4), (4.1), (4.4)

$$
\left|\frac{\partial \lambda_{1}(x, \bar{t})}{\partial \nu}\right| \leq C \frac{r}{r_{0}^{2}}
$$

which implies

$$
\int_{\tilde{\Gamma}_{1, r}}\left|\frac{\partial \lambda_{1}(x, \bar{t})}{\partial \nu}\right| d \sigma \leq C r r_{0}^{n-3},
$$

where $C$ is a constant depending on the a priori data, except $\Phi_{0}, \Phi_{1}$. Let us now consider $x \in \tilde{\Gamma}_{2, r}$. As before there exists $y \in \partial \Omega_{1} \backslash \Sigma$ such that $|y-x|=$ $\operatorname{dist}\left(x, \partial \Omega_{2}\right) \leq \xi_{2} r$. Since $\frac{\partial \lambda_{2}}{\partial \nu}=0$ on $I_{2} \times\left[t_{1}, T\right]$, we have that

$$
\left|\frac{\partial \lambda_{1}(x, \bar{t})}{\partial \nu}\right| \leq\left|\nabla \lambda_{1}(x, \bar{t})-\nabla \lambda_{2}(x, \bar{t})\right|+\left|\nabla \lambda_{2}(x, \bar{t})-\nabla \lambda_{2}(y, \bar{t})\right| .
$$

Thus we get

$$
\int_{\tilde{\Gamma}_{2, r}}\left|\frac{\partial \lambda_{1}(x, \bar{t})}{\partial \nu}\right| d \sigma \leq C r_{0}^{n-3} r+\int_{\tilde{\Gamma}_{2, r}}\left|\nabla \lambda_{1}(x, \bar{t})-\nabla \lambda_{2}(x, \bar{t})\right| d \sigma,
$$

where $C$ is a constant depending on the a priori data, except $\Phi_{0}, \Phi_{1}$.

Let us consider the integral on the right hand side of (4.15). First observe that

$$
\begin{aligned}
& \nabla \lambda_{1}-\nabla \lambda_{2} \\
= & \frac{1}{\left(u_{1} u_{2}\right)^{2}}\left\{u_{1} u_{2}^{2}\left(\nabla \widetilde{u}_{1}-\nabla \widetilde{u}_{2}\right)-\tilde{u}_{1} u_{2}^{2}\left(\nabla u_{1}-\nabla u_{2}\right)\right. \\
& \left.+u_{2}^{2} \nabla \tilde{u}_{2}\left(u_{1}-u_{2}\right)-u_{2}^{2} \nabla u_{2}\left(\tilde{u}_{1}-\tilde{u}_{2}\right)+\left(u_{2}^{2}-u_{1}^{2}\right)\left(u_{2} \nabla \tilde{u}_{2}-\tilde{u}_{2} \nabla u_{2}\right)\right\} .
\end{aligned}
$$

Now labeling $w=u_{1}-u_{2}, \tilde{w}=\tilde{u}_{1}-\tilde{u}_{2}$ and taking into account (4.15) and (4.16), we get

$$
\begin{aligned}
& \int_{\tilde{\Gamma}_{2, r}}\left|\frac{\partial \lambda_{1}(x, \bar{t})}{\partial \nu}\right| d \sigma \\
\leq & C r_{0}^{n-2}\left(\frac{r}{r_{0}}+r_{0} \max _{x \in \tilde{V}_{r}}|\nabla \tilde{w}(x, \bar{t})|+r_{0} \max _{x \in \bar{V}_{r}}|\nabla w(x, \bar{t})|\right. \\
& \left.+\max _{x \in \bar{V}_{r}}|\tilde{w}(x, \bar{t})|+\max _{x \in \tilde{V}_{r}}|w(x, \bar{t})|\right),
\end{aligned}
$$

where $C$ is a constant depending on the a priori data, except $\Phi_{0}, \Phi_{1}$. To evaluate the maximum of $w, \tilde{w}$ and their gradients we can proceed as in [8, Proposition 5.3]. 
Let us briefly sketch the main items of this proof for $\nabla w$, for instance. Suppose $\max _{x \in \tilde{V}_{r}}|\nabla w(x, \bar{t})|=|\nabla w(\bar{x}, \bar{t})|=\|\nabla w(\cdot, \bar{t})\|_{L^{\infty}\left(B_{r}(\bar{x})\right)}$, with $\bar{x} \in \overline{\tilde{V}}_{r}$. By interpolation inequality (see [8, A2, page 553, with $\alpha=1 / 2]$ ), (2.2c) and (2.4), we have

$$
\|\nabla w(\cdot, \bar{t})\|_{L^{\infty}\left(B_{r}(\bar{x})\right.} \leq \frac{C r_{0}}{r^{1+\frac{n}{2(n+2)}}}\left(\int_{B_{r}(\bar{x})} w^{2}(x, \bar{t}) d x\right)^{\frac{1}{2(n+2)}},
$$

where $C$ depends on the a priori data, except $\Phi_{0}, \Phi_{1}$. Now, in order to apply Theorem 4.1 , we estimate $\|w\|_{H^{3 / 2,1 / 2}\left(\Sigma \times\left(t_{1}, T\right)\right)}=\left\|u_{1}-u_{2}\right\|_{H^{3 / 2,1 / 2}\left(\Sigma \times\left(t_{1}, T\right)\right)}$ in terms of $\left\|u_{1}-u_{2}\right\|_{L^{2}\left(\Sigma \times\left(t_{1}, T\right)\right)}$ and of the a priori data. The functions $w, w_{t}, w_{t t}$ satisfy the problem

$$
\begin{cases}z_{t}-\Delta z=0, & \text { in } G \times(0, T), \\ z=0, & \text { on } G \times\{0\}, \\ \frac{\partial z}{\partial \nu}=0, & \text { on } A \times(0, T) .\end{cases}
$$

Hence, recalling that $A^{r_{0}}=\left\{x \in A: \operatorname{dist}(x, I)>r_{0}\right\}$ and $\operatorname{denoting} \mathcal{U}^{r_{0} / 8}=\{x \in$ $G$ : $\left.\operatorname{dist}\left(x, A^{r_{0}}\right) \leq r_{0} / 8\right\}$, we may apply the local bound estimates [15] and obtain

$$
\begin{gathered}
\left\|w_{t}\right\|_{L^{\infty}\left(\mathcal{U}^{r_{0} / 8} \times(0, T)\right)} \leq C\|g\|_{H^{1 / 2,1 / 4}(A \times(0, T))} \leq C\|g\|_{C^{1,1}(A \times(0, T))}, \\
\left\|w_{t t}\right\|_{L^{\infty}\left(\mathcal{U}^{r_{0} / 8} \times(0, T)\right)} \leq C\|g\|_{H^{1 / 2,1 / 4}(A \times(0, T))} \leq C\|g\|_{C^{1,1}(A \times(0, T))},
\end{gathered}
$$

where $C$ depends on $r_{0}^{2} T^{-1}, L$. We may also think of $w(\cdot, t)$, for a fixed $t \in(0, T)$, as the solution of the elliptic problem

$$
\begin{cases}\Delta w(x, t)=w_{t}(x, t), & \text { in } G, \\ \frac{\partial w}{\partial \nu}(x, t)=0, & \text { on } A,\end{cases}
$$

and, similarly, we may think of $w_{t}(\cdot, t)$, for a fixed $t \in(0, T)$, as the solution of the elliptic problem

$$
\begin{cases}\Delta w_{t}(x, t)=w_{t t}(x, t) & \text { in } G, \\ \frac{\partial w_{t}}{\partial \nu}(x, t)=0, & \text { on } A .\end{cases}
$$

By $L^{p}$ regularity estimates (see [12]), by (4.18), (4.19), by trace inequalities and by the immersion of $W_{p}^{2-1 / p}$ in $H^{2-1 / p}$ for $p>2$, we have

$$
\sup _{t \in[0, T]}\left(\|w(\cdot, t)\|_{H^{2-1 / p}(\Sigma)}+\left\|w_{t}(\cdot, t)\right\|_{H^{2-1 / p}(\Sigma)}\right) \leq C\|g\|_{C^{1,1}(A \times(0, T))},
$$

for any $p>2$, where $C$ depends on $L, r_{0}^{2} / T$ only. Therefore

$$
\|w\|_{H^{\alpha, \alpha / 2}(\Sigma \times(0, T))} \leq C\|g\|_{C^{1,1}(A \times(0, T))},
$$

with $\alpha=2-1 / p>3 / 2$, where $C$ depends on $L, r_{0}^{2} / T$ only. By interpolation we have

$$
\|w\|_{H^{3 / 2,3 / 4}(\Sigma \times(0, T))} \leq C\|w\|_{H^{\alpha, \alpha / 2}(\Sigma \times(0, T))}^{1-\theta}\|w\|_{L^{2}(\Sigma \times(0, T))}^{\theta},
$$

where $\theta$ is given by $(1-\theta) \alpha=3 / 2$ (see [18). By (2.2c), (2.5), (4.20), (4.21), choosing $p=4$ we have

$$
\|w\|_{H^{3 / 2,3 / 4}(\Sigma \times(0, T))} \leq C \varepsilon^{1 / 7}
$$


where $C$ depends on $L, E, r_{0}^{2} / T$ only. Let $P_{1} \in \Sigma$ be such that $\partial \Omega \cap B_{r_{0}}\left(P_{1}\right) \subset \Sigma$. By Theorem 4.1 and (4.22), we get

$$
\|w(\cdot, \bar{t})\|_{L^{2}\left(B_{\bar{\theta} r_{0}}\left(P_{2}\right)\right)} \leq C \varepsilon^{\bar{\tau} / 7},
$$

where $P_{2}, \bar{\theta}, \bar{\tau}$ are as in the above theorem, $C \geq 1$ depends on $L, E, r_{0}^{2} / T$ only and $\bar{t}$ is the point in (4.12). Now, let $\sigma$ be an arc in $\tilde{V}_{r}$ joining $\bar{x}$ with $P_{2}$ (since $\bar{\theta} r_{0}>r$, the point $\left.P_{2} \in \tilde{V}_{r}\right)$. Let us define $\left\{x_{i}\right\}, i=1,2, \ldots, s$, as follows: $x_{1}=P_{2}$, $x_{i+1}=\sigma\left(\eta_{i}\right)$, where $\eta_{i}=\max \left\{\eta:\left|\sigma(\eta)-x_{i}\right|=2 \bar{\theta} r\right\}$ if $\left|x_{i}-\bar{x}\right|>2 \bar{\theta} r$; otherwise $i=s$ and stop the process. By construction, the balls $B_{\bar{\theta} r}\left(x_{i}\right)$ are pairwise disjoint, $\left|x_{i+1}-x_{i}\right|=2 \bar{\theta} r$, for $i=1,2, \ldots, s-1,\left|x_{s}-\bar{x}\right| \leq 2 \bar{\theta} r$. Notice that $s \leq \frac{C}{r^{n}}$, where $C$ depends on $M$ only. By an iterated application of the two-sphere one-cylinder inequality over the chain of balls $B_{r_{1}}\left(x_{i}\right)$, for $r_{1}=\frac{\bar{\theta}}{26 \sqrt{2}} r$, by (2.2c), (2.4), (4.8), (4.14), (4.15), (4.7), (4.12), (4.23), (4.17), we get, for $t_{1} \leq t \leq T$,

$$
\int_{\Omega_{1} \backslash G} u_{1}^{2}(x, t) \lambda_{1}^{2}(x, t) d x \leq C r_{0}^{n}\left(\frac{r}{r_{0}}+\left(\frac{r_{0}}{r}\right)^{\frac{4 n+10}{3 n+6}} \varepsilon^{\frac{2 \tau^{s}+1}{7(3 n+6)}}\right),
$$

where $C$ depends on the a priori data, except $\Phi_{0}$. With a suitable choice of $r=r(\varepsilon)$, by standard arguments we get the thesis.

Proof of Proposition 3.2. By the use of the divergence theorem over the Lipschitz domains $\Omega_{1}$ and $G$ and the same arguments based on the application of the Gronwall inequality developed in Proposition 3.1, we have that

$$
\int_{\Omega_{1} \backslash \bar{G}} \lambda_{1}^{2}(x, t) d x \leq C r_{0}^{2} \int_{\partial\left(\Omega_{1} \backslash \bar{G}\right)}\left|\partial_{\nu} \lambda_{1}(x, \bar{t})\right| d \sigma,
$$

where $\bar{t} \in\left[t_{1}, t\right]$ is the point where $\int_{\partial\left(\Omega_{1} \backslash \bar{G}\right)}\left|\partial_{\nu} \lambda_{1}(x, t)\right| d \sigma$ achieves its maximum, and $C>0$ is a constant depending on the a priori data only. Moreover, we observe that $\partial\left(\Omega_{1} \backslash \bar{G}\right) \subset\left(\partial \Omega_{1} \backslash A\right) \cup\left(\partial \Omega_{2} \cap \partial G \backslash U_{A}^{\frac{r_{0}}{2}}\right)$, where $U_{A}^{\frac{r_{0}}{2}}=\left\{x \in \bar{G}, \operatorname{dist}\left(x, A^{r_{0}}\right) \leq \frac{r_{0}}{2}\right\}$.

Since $\partial_{\nu} \lambda_{1}=0$ on $\partial \Omega_{1} \backslash A$ and since $\partial_{\nu} \lambda_{2}=0$ on $\partial \Omega_{2} \backslash A$, we found that

$$
\begin{aligned}
\int_{\Omega_{1} \backslash \bar{G}} \lambda_{1}^{2}(x, t) d x & \leq C r_{0}^{2} \int_{\left(\partial \Omega_{2} \cap \partial G\right) \backslash U_{A}^{\frac{r_{0}}{2}}}\left|\partial_{\nu} \lambda_{1}(x, \bar{t})-\partial_{\nu} \lambda_{2}(x, \bar{t})\right| d x \\
& \leq C_{1} r_{0}^{n+1} \max _{\partial G}\left|\nabla \lambda_{1}(x, \bar{t})-\nabla \lambda_{2}(x, \bar{t})\right|,
\end{aligned}
$$

where $C_{1}$ is a constant depending on the a priori data only. By the same argument of Proposition 3.1 and using the same notation we get

$$
\int_{\Omega_{1} \backslash \bar{G}} \lambda_{1}^{2}(x, t) d x \leq C_{2} r_{0}^{n}\left(\max _{\partial G}|w|+\max _{\partial G}|\tilde{w}|+r_{0} \max _{\partial G}|\nabla w|+r_{0} \max _{\partial G}|\nabla \tilde{w}|\right),
$$

where $C_{2}$ is a constant depending on the a priori data only.

In order to control the maximum of $w, \tilde{w}$ and their gradients we argue as in Proposition 5.4 of [8]. We carry out our analysis for the term $\nabla w$, the other cases being analogous. Let $P_{1} \in \Sigma$ be such that $\partial \Omega \cap B_{r_{0}}\left(P_{1}\right) \subset \Sigma$. Now by Theorem 4.1, arguing as to achieve (4.23), we may obtain

$$
\|w(\cdot, \bar{t})\|_{L^{2}\left(B_{\bar{\theta} r_{0}}\left(P_{2}\right)\right)} \leq \tilde{C} \varepsilon^{\frac{\bar{\tau}}{7}}
$$

where $P_{2}=P_{1}-\bar{\theta} r_{0} \nu, \bar{\theta}, \bar{\tau}$ are as in the above theorem, and $\tilde{C}>1$ depends on $L, E, \frac{r_{0}^{2}}{T}$. 
Given $z \in \mathbb{R}^{n}, \xi \in \mathbb{R}^{n},|\xi|=1, \theta>0, r>0$, we shall denote by

$$
C(z, \xi, \theta, r)=\left\{x \in \mathbb{R}^{n}: \frac{(x-z) \cdot \xi}{|x-z|}>\cos (\theta),|x-z|<r\right\}
$$

the intersection of the ball $B_{r}(z)$ and the open cone having vertex $z$, axis in the direction $\xi$ and width $2 \theta$. Since $\partial G$ is of Lipschitz class with constant $r_{1}, L_{1}$ for any $z \in \partial G$, there exists $\xi \in \mathbb{R}^{n},|\xi|=1$, such that $C\left(z, \xi, \theta, r_{1}\right) \subset G$, where $\theta=\arctan \frac{1}{L_{1}}$.

Let $(\bar{x}, \bar{t}) \in \partial G$ be such that $|\nabla w(\bar{x}, \bar{t})|=\|\nabla w(\cdot, \bar{t})\|_{L^{\infty}(\partial G)}$. Now dealing as in Proposition 5.4 in [8], we combine the inequality (4.26) with an iterated use of the two-sphere and one-cylinder inequality (Theorem 3.7) within the cone $C\left(\bar{x}, \xi, \theta, r_{1}\right)$ obtaining the following estimate:

$$
\|w(\cdot, \bar{t})\|_{L^{2}\left(B_{\rho_{k(r)}}\left(x_{k(r)}\right)\right)}^{2} \leq C\left(1+\frac{T^{2}}{\rho_{k(r)}^{4}}\right)^{1-\bar{\tau}^{k(r)-1}} \varepsilon^{\beta_{1} \bar{\tau}^{k(r)-1}},
$$

with

$$
\begin{aligned}
& \rho_{0}=a_{1} \sin \theta, \quad \rho_{k}=\chi \rho_{k-1}, \quad d_{1}=a_{1}(1-\sin \theta), \\
& \frac{\left|\log \frac{r}{d_{1}}\right|}{|\log \chi|} \leq k(r)-1 \leq \frac{\left|\log \frac{r}{d_{1}}\right|}{|\log \chi|}+1,
\end{aligned}
$$

where $0<\bar{\tau}<1,0<\beta_{1}<1,0<\chi<1, a_{1}>0$ are positive constants depending on the a priori data only and where $x_{k(r)}$ is a point lying on the axis $\xi$ of the cone $C\left(\bar{x}, \xi, \theta, r_{1}\right)$ at a distance $\chi^{k(r)-1} \cdot d_{1}+\rho_{k(r)}$ from $\bar{x}$ with $0<r<d_{1}$. By the interpolation inequality (4.17) recalled in the proof of Proposition 3.1 and the definition of $\rho_{k(r)}$, we have that (4.28) leads to

$$
\|\nabla w(\cdot, \bar{t})\|_{L^{\infty}\left(B_{\rho_{k(r)}}\left(x_{k(r)}\right)\right)} \leq \frac{C}{r_{0}} \chi^{\beta_{3}(1-k(r))} \varepsilon^{\beta_{2} \bar{\tau}^{k(r)-1}},
$$

where $C>0,0<\beta_{2}<1,0<\beta_{3}<1$ are constants depending on the a priori data only. We consider the point $x_{r}=\bar{x}+r \xi$. We have that $x_{r} \in B_{\rho_{k(r)}}\left(x_{k(r)}\right)$. From (4.29) and from the $C^{1, \alpha}$ regularity of $w$ we have that

$$
|\nabla w(\bar{x}, \bar{t})| \leq \frac{C}{r_{0}}\left(\left(\frac{r}{r_{0}}\right)^{\alpha}+\chi^{\beta_{3}(1-k(r))} \varepsilon^{\beta_{2} \bar{\tau}^{k(r)-1}}\right),
$$

where $C>0$ depends on the a priori data only. Minimizing with respect to $r$ we obtain the desired estimate.

Let us now consider the proofs of Proposition 3.3 . For this purpose we need a Harnack inequality in the interior and its version at the boundary. The first tool can be found in [19]; we recall it for the reader's convenience.

Let $A(x, t)$ be a symmetric matrix, $A(x, t) \in L^{\infty}\left(\mathbb{R}^{n+1}\right)$, such that

$$
\lambda^{-1}|\xi|^{2} \leq A(x, t) \xi \cdot \xi \leq \lambda|\xi|^{2}, \quad \forall(x, t) \in \mathbb{R}^{n+1}, \forall \xi \in \mathbb{R}^{n},
$$

with $\lambda \geq 1$.

Theorem 4.2 (Harnack inequality in the interior; 19], Theorem 2, page 104). Let $A(x, t)$ be a symmetric matrix, $A(x, t) \in L^{\infty}\left(\mathbb{R}^{n+1}\right)$, satisfying (4.31); let $R>0$, $d \in(0, R), T>0$. Let $u(x, t)>0$ be a weak solution of the equation

$$
\operatorname{div}(A(x, t) \nabla u)-\partial_{t} u=0, \quad \text { in } B_{R} \times(0, T] .
$$


Then, if $(y, s),(x, t) \in B_{R-d} \times(0, T], d^{2} \leq s<t \leq T$, we have

$$
u(y, s) \leq C_{1} u(x, t),
$$

where $C_{1}=\exp c_{0}\left\{\frac{|x-y|^{2}}{t-s}+\frac{t-s}{d^{2}}+1\right\}$, with $c_{0}$ depending only on $\lambda$ and $n$.

Proposition 4.3 (Harnack inequality at the boundary). Let $\Omega \subseteq \mathbb{R}^{n}$ be a bounded domain, and let $\partial \Omega$ be of class $C^{1, \alpha}$ with constants $L, r_{0}$. Suppose $0 \in \partial \Omega$ and let

$$
\Gamma:=\partial \Omega \cap B_{r_{0}} .
$$

Let $\gamma(x, t) \in L^{\infty}(\Gamma \times(0, T])$ such that

$$
0 \leq \gamma(x, t) \leq \bar{\gamma} r_{0}^{-1} \quad \text { on } \Gamma \times(0, T] \text { a.e. }
$$

Let $u(x, t)>0$ be a weak solution to the problem

$$
\begin{cases}\operatorname{div}(A(x, t) \nabla u)-\partial_{t} u=0, & \left(B_{r_{0}} \cap \Omega\right) \times(0, T], \\ A(x, t) \nabla u \cdot \nu+\gamma(x, t) u=0, & \Gamma \times(0, T] .\end{cases}
$$

Let $T_{1} \in(0, T)$; then there exists $\bar{\theta}, 0<\bar{\theta}<\frac{1}{2}$, depending on $L$, such that if

$$
0<r \leq \min \left\{\bar{\theta} r_{0}, \sqrt{T-T_{1}}, \sqrt{T_{1}}\right\},
$$

we get

$$
\max _{\overline{B_{r / 4} \cap \Omega} \times\left[T_{1}, T_{1}+\frac{r^{2}}{3}\right]} u(x, t) \leq C_{1} \underset{\min _{B_{r / 4} \cap \Omega} \times\left[T_{1}+\frac{2 r^{2}}{3}, T_{1}+r^{2}\right]}{ } u(x, t),
$$

where $C_{1}$ depends only on $\lambda, \bar{\gamma}, L$ and $n$.

The proof of Proposition 4.3 is postponed to Section 5.

Remark 4.4. Let us observe that the result of the above Proposition 4.3 holds true, weakening the regularity assumptions on the boundary $\left(C^{0,1}\right.$ instead of $\left.C^{1, \alpha}\right)$.

Proof of Proposition 3.3. Set $y_{0} \in A_{2 r_{0}}$ and set $t_{2}:=\frac{t_{1}}{2}$. First we prove that there exists $\delta \in\left(0, \frac{1}{4}\right)$ and $K_{0}>0$ such that if $y_{1}=y_{0}-2 r_{0} \delta \nu$, we get

$$
u\left(y_{1}, t_{2}\right) \geq K_{0},
$$

where $\delta, K_{0}$ depend on $\Phi_{1}, E, M, L, \bar{\gamma}$.

In fact, by the Lagrange theorem, we have, for $\tau>0$,

$$
u\left(y_{0}-\tau \nu, t_{2}\right)-u\left(y_{0}-2 \tau \nu, t_{2}\right)=\nabla u\left(y_{0}-\eta \nu, t_{2}\right) \cdot \nu \tau,
$$

where $\eta \in(\tau, 2 \tau)$. By (2.4) we get

$$
\nabla u\left(y_{0}-\eta \nu, t_{2}\right) \cdot \nu \geq \nabla u\left(y_{0}, t_{2}\right) \cdot \nu-C_{0}\|g\|_{C^{0,1}(A \times[0, T])} \frac{\eta^{\alpha}}{r_{0}^{1+\alpha}} .
$$

Inserting (4.33) in (4.32), by (2.2f), (2.2c), we obtain

$$
\begin{gathered}
u\left(y_{0}-\tau \nu, t_{2}\right) \geq \nabla u\left(y_{0}, t_{2}\right) \cdot \nu \tau-C_{0} E \frac{\eta^{\alpha}}{r_{0}^{1+\alpha}} \tau \\
\geq g\left(y_{0}, t_{2}\right) \tau-C_{0} E \frac{(2 \tau)^{\alpha}}{r_{0}^{1+\alpha}} \tau \geq \frac{\tau}{r_{0}}\left(\Phi_{1}-C_{0} E\left(\frac{2 \tau}{r_{0}}\right)^{\alpha}\right) .
\end{gathered}
$$

Choosing $\tau=\bar{\tau}=\frac{r_{0}}{2} \min \left\{\left(\frac{\Phi_{1}}{2 C_{0} E}\right)^{1 / \alpha}, 1\right\}$, we conclude that

$$
u\left(y_{0}-\bar{\tau} \nu, t_{2}\right) \geq \frac{\Phi_{1}}{2}\left(\frac{\Phi_{1}}{2 C_{0} E}\right)^{1 / \alpha}=: K_{0} .
$$


Defining $2 r_{0} \delta=\bar{\tau}$, that is,

$$
\delta=\min \left\{\frac{1}{4}, \frac{1}{4}\left(\frac{\Phi_{1}}{2 C_{0} E}\right)^{1 / \alpha}\right\}
$$

we get (4.35) .

In order to estimate from below $u(x, t)$ for $(x, t) \in \bar{\Omega} \times\left[t_{1}, T\right]$, we proceed in two steps. Let $\eta \in(0, \delta]$ to be determined later, while $\delta$ is defined in (4.40). In the first step we get such an estimate for $(x, t) \in \Omega_{\eta r_{0}} \times\left[\frac{3}{4} t_{1}, T\right]$, making use of the Harnack inequality in the interior. In the second step we achieve such an estimate for $(x, t)$ $\in\left(\bar{\Omega} \backslash \Omega_{\eta r_{0}}\right) \times\left[t_{1}, T\right]$, making use of the Harnack inequality at the boundary and of the result of the first step.

Recall that

$$
\Omega_{\eta r_{0}}=\left\{x \in \Omega: \operatorname{dist}(x, \partial \Omega)>\eta r_{0}\right\} .
$$

$1^{\text {st }}$ step. Let $x \in \Omega_{\eta r_{0}}, t \in\left[\frac{3}{4} t_{1}, T\right]$ and let $\sigma:[0,1] \rightarrow \Omega_{\eta r_{0}}$ be a curve joining $y_{1}$ to $x$. As usual, we construct a chain of balls $B \frac{\eta r_{0}}{4}\left(y_{i}\right), i=1,2, \ldots, N$. Let us define $\left\{y_{i}\right\}, i=1,2, \ldots, N$, as follows: $y_{1}=y_{1}, y_{i+1}=\sigma\left(s_{i}\right)$, where $s_{i}=$ $\max \left\{s:\left|\sigma(s)-y_{i}\right|=\frac{\eta r_{0}}{2}\right\}$ if $\left|y_{i}-x\right|>\frac{\eta r_{0}}{2}$; otherwise $i=N$ and stop the process. By construction the balls $B_{\frac{\eta r_{0}}{4}}\left(y_{i}\right)$ are pairwise disjoint, $\left|y_{i+1}-y_{i}\right|=\frac{\eta r_{0}}{2}$, for $i=1, \ldots, N-1,\left|y_{N}-x\right| \leq \frac{\eta r_{0}}{2}$. Let us divide the interval $\left[t_{2}, t\right]$, where $t_{2}=\frac{t_{1}}{2}$, into $N$ equal parts $h=\frac{t-t_{2}}{N}$ by the points $\tau_{j}$, where $\tau_{1}=t_{2}, \tau_{j}=t_{2}+(j-1) h$, $j=2, \ldots, N+1$.

Now we apply, for $j \leq N-1$ fixed, the Harnack inequality in the interior (Theorem 4.2) in the cylinder

$$
B_{\eta r_{0}}\left(y_{j}\right) \times\left(0, \tau_{j+1}\right] .
$$

Since $\left(y_{j}, \tau_{j}\right),\left(y_{j+1}, \tau_{j+1}\right) \in B_{\frac{\eta r_{0}}{2}}\left(y_{j}\right) \times\left(0, \tau_{j+1}\right]$ and $\tau_{j} \geq t_{2}$, denoting by

$$
d_{1}=\min \left\{\sqrt{t_{2}}, \frac{\eta r_{0}}{2}\right\},
$$

we get

$$
u\left(y_{j}, \tau_{j}\right) \leq\left\{\exp c\left[\frac{\left|y_{j}-y_{j+1}\right|^{2}}{\tau_{j+1}-\tau_{j}}+\frac{\tau_{j+1}-\tau_{j}}{d_{1}^{2}}+1\right]\right\} u\left(y_{j+1}, \tau_{j+1}\right),
$$

where $c$ is an absolute constant.

Recalling $t \geq \frac{3}{4} t_{1}$, we estimate

$$
\frac{\left|y_{j}-y_{j+1}\right|^{2}}{\tau_{j+1}-\tau_{j}}+\frac{\tau_{j+1}-\tau_{j}}{d_{1}^{2}}+1 \leq \frac{\left(\eta r_{0}\right)^{2} N}{t_{1}}+\frac{T-\frac{t_{1}}{2}}{d_{1}^{2}}+1=: K_{1} .
$$

Iterating the process we get, by (4.39),

$$
K_{0} e^{-c(N-1) K_{1}} \leq u\left(y_{N}, \tau_{N}\right) .
$$

We again apply Theorem 4.2 in

$$
B_{\eta r_{0}}\left(y_{N}\right) \times(0, t] .
$$

Since $y_{N}, x \in B_{\frac{3}{4} \eta r_{0}}\left(y_{N}\right)$, denoting

$$
d_{2}=\min \left\{\sqrt{t_{2}}, \frac{\eta r_{0}}{4}\right\}
$$


we get

$$
e^{-c^{\prime} K_{2}} u\left(y_{N}, \tau_{N}\right) \leq u(x, t)
$$

where

$$
K_{2}=\frac{N\left(\eta r_{0}\right)^{2}}{t_{1}}+\frac{T-\frac{t_{1}}{2}}{\min \left\{16 t_{2},\left(\eta r_{0}\right)^{2}\right\}}+1,
$$

with $c^{\prime}$ an absolute constant.

By (4.43), (4.45), we obtain

$$
K_{0} e^{-c(N-1) K_{1}-c^{\prime} K_{2}} \leq u(x, t) .
$$

Since $N \leq \frac{|\Omega|}{c_{n}\left(\frac{\eta_{0}}{4}\right)^{n}} \leq c_{n}^{\prime} \frac{M}{\eta^{n}}$, by (4.47) we can conclude that

$$
K_{3} \leq u(x, t), \quad \text { for } x \in \Omega_{\eta r_{0}}, t \in\left[\frac{3}{4} t_{1}, T\right],
$$

where

$$
K_{3}=K_{0} \exp \left\{-c\left[\frac{r_{0}^{2}}{t_{1}} \frac{M^{2}}{\eta^{2(n-1)}}+\frac{M}{\eta^{n}}+\frac{T-\frac{t_{1}}{2}}{\min \left\{2 t_{1},\left(\eta r_{0}\right)^{2}\right\}}+1\right]\right\},
$$

with $c$ an absolute constant.

$2^{\text {nd }}$ step. Let $(\bar{x}, \bar{t}) \in\left(\bar{\Omega} \backslash \Omega_{\eta r_{0}}\right) \times\left[t_{1}, T\right]$. Let $x_{0} \in \partial \Omega$ such that

$$
\left|\bar{x}-x_{0}\right|=\min _{y \in \partial \Omega}|y-\bar{x}|,
$$

which implies $\left|\bar{x}-x_{0}\right| \leq \eta r_{0}$. Suppose $x_{0}=0$. Now we apply the Harnack inequality at the boundary; with reference to the notation of Proposition 4.3, we set

$$
\begin{aligned}
r & =8 \eta r_{0}, \\
T_{1} & =\bar{t}-r^{2}, \\
T & =\bar{t} .
\end{aligned}
$$

We now have to satisfy condition (4.34) of Proposition 4.3 and the condition $T_{1}=$ $\bar{t}-r^{2} \geq \frac{3}{4} t_{1}$, since estimate (4.48) holds for $t \in\left[\frac{3}{4} t_{1}, T\right]$.

The second condition implies, since $\bar{t} \geq t_{1}$,

$$
\eta \leq \frac{\sqrt{t_{1}}}{16 r_{0}} \text {. }
$$

The first condition is

$$
8 \eta r_{0} \leq \min \left\{\bar{\theta} r_{0}, 8 \eta r_{0}, \sqrt{\frac{3 t_{1}}{4}}\right\}
$$

where $\bar{\theta}$ as in Proposition 4.3; that is,

$$
\eta \leq \min \left\{\frac{\bar{\theta}}{8}, \frac{\sqrt{3 t_{1}}}{16 r_{0}}\right\} .
$$

Taking into account $\eta \in(0, \delta]$, by (4.52) we can choose

$$
\eta=\min \left\{\delta, \frac{\bar{\theta}}{8}, \frac{\sqrt{t_{1}}}{16 r_{0}}\right\} .
$$


Applying the Harnack inequality at the boundary, by (4.48) and since $\bar{B}_{2 \eta r_{0}} \cap \bar{\Omega}_{\eta r_{0}} \neq$ $\emptyset$, we obtain

$$
\begin{aligned}
& K_{3} \leq \max _{\left(\bar{B}_{2 \eta r_{0}} \cap \bar{\Omega}\right) \times\left[\bar{t}-r^{2}, \bar{t}-\frac{2}{3} r^{2}\right]} u(x, t) \\
& \leq C_{1} \min _{\left(\bar{B}_{2 \eta r_{0}} \cap \bar{\Omega}\right) \times\left[\bar{t}-\frac{r^{2}}{3}, \bar{t}\right]} u(x, t) \leq C_{1} u(\bar{x}, \bar{t}),
\end{aligned}
$$

where $C_{1}$ depends on $L, \bar{\gamma}$.

Finally, collecting (4.55), 4.48), we can conclude that

$$
\min \left(\frac{K_{3}}{C_{1}}, K_{3}\right) \leq u(x, t), \quad \text { in } \bar{\Omega} \times\left[t_{1}, T\right],
$$

where $K_{3}$ is defined in (4.49), $\eta$ is defined in (4.54) and $C_{1}$ depends on $L, \bar{\gamma}$. We get the thesis, denoting it by $c_{0}=\min \left(\frac{K_{3}}{C_{1}}, K_{3}\right)$, where $c_{0}$ depends on the a priori data except $\Phi_{0}$.

In order to prove Proposition 3.4. we need a technical lemma. Let us introduce the following notation. We shall denote by $b_{0}, b_{1}$ two positive constants such that

$$
b_{0} \leq u(x, t) \leq b_{1} \quad \forall(x, t) \in \bar{\Omega} \times\left[t_{1}, T\right]
$$

(by (3.6) we can take $b_{0}=c_{0}$, whereas the existence of $b_{1}$ is guaranteed by (2.2c) and (2.4) $)$.

Lemma 4.5. Let the hypothesis of Theorem 2.5 be satisfied. We have that, for $i=1,2$,

$$
\begin{gathered}
\int_{\Omega} \lambda_{i}^{2}(x, t) d x+b_{0}^{2} \int_{t_{1}}^{t} \int_{\Omega_{i}}\left|\nabla \lambda_{i}(x, t)\right|^{2} d x d t \\
(4.56) \leq C_{0} r_{0} b_{0}^{2}\left(1+e^{b_{0}^{2} t r_{0}^{-2}}\right) \int_{t_{1}}^{t} \int_{\partial \Omega_{i}}\left|u_{i}^{2}(x, \tau) \partial_{\nu} \lambda_{i}(x, \tau)\right|^{2} d \sigma d \tau, \quad \forall t \in\left[t_{1}, T\right],
\end{gathered}
$$

where $C_{0}>0$ is a constant depending on the a priori data only.

Proof. For the sake of brevity, we shall denote along the proof $h_{i}=u_{i}^{2} \partial_{\nu} \lambda_{i}$ and we drop all the subindexes $i$. By the weak formulation of problem (3.3) satisfied by $\lambda$, we obtain that

$$
\begin{aligned}
& \frac{1}{2} \int_{t_{1}}^{t} \int_{\Omega}\left(\lambda^{2}\right)_{\tau} d x d \tau+\int_{t_{1}}^{t} \int_{\Omega} u^{2}|\nabla \lambda|^{2} d x d \tau \\
= & \int_{t_{1}}^{t} \int_{\partial \Omega} h \lambda d \sigma d \tau .
\end{aligned}
$$

By the Hölder inequality, we get

$$
\begin{aligned}
& \frac{1}{2} \int_{\Omega} \lambda^{2} d x+\int_{t_{1}}^{t} \int_{\Omega} u^{2}|\nabla \lambda|^{2} d x d \tau \\
\leq & \left(\int_{t_{1}}^{t} \int_{\partial \Omega} h^{2} d \sigma d \tau\right)^{\frac{1}{2}}\left(\int_{t_{1}}^{t} \int_{\partial \Omega} \lambda^{2} d \sigma d \tau\right)^{\frac{1}{2}} .
\end{aligned}
$$

We recall the following trace inequality (see [17]):

$$
\|\lambda(\cdot, t)\|_{L^{2}(\partial \Omega)} \leq C\left(r_{0}^{-1 / 2}\|\lambda(\cdot, t)\|_{L^{2}(\Omega)}+r_{0}^{1 / 2}\|\nabla \lambda(\cdot, t)\|_{L^{2}(\Omega)}\right),
$$


where $C>0$ is a constant depending on $r_{0}$ and $L$ only. Raising to the square the latter and integrating over the interval $\left[t_{1}, t\right]$, we have that

$$
\int_{t_{1}}^{t} \int_{\partial \Omega} \lambda^{2} d \sigma d \tau \leq C\left(r_{0}^{-1} \int_{t_{1}}^{t} \int_{\Omega} \lambda^{2} d x d \tau+r_{0} \int_{t_{1}}^{t} \int_{\Omega}|\nabla \lambda|^{2} d x d \tau\right) .
$$

Plugging the above estimate in the right hand side of (4.58) and using the Young inequality we get, for $\delta>0$,

$$
\begin{aligned}
& \frac{1}{2} \int_{\Omega} \lambda^{2} d x+b_{0}^{2} \int_{t_{1}}^{t} \int_{\Omega}|\nabla \lambda|^{2} d x d \tau \\
& \leq \frac{C r_{0}}{\delta} \int_{t_{1}}^{t} \int_{\partial \Omega} h^{2} d \sigma d \tau+\delta r_{0}^{-2} \int_{t_{1}}^{t} \int_{\Omega} \lambda^{2} d x d \tau+\delta \int_{t_{1}}^{t} \int_{\Omega}|\nabla \lambda|^{2} d x d \tau, \quad \forall t \in\left[t_{1}, T\right] .
\end{aligned}
$$

Choosing $\delta=\frac{1}{2} b_{0}^{2}$ we obtain that

$$
\begin{aligned}
& \int_{\Omega} \lambda^{2} d x \\
\leq & C r_{0} b_{0}^{2} \int_{t_{1}}^{t} \int_{\partial \Omega} h^{2} d \sigma d \tau+b_{0}^{2} r_{0}^{-2} \int_{t_{1}}^{t} \int_{\Omega} \lambda^{2} d x d \tau, \quad \forall t \in\left[t_{1}, T\right] .
\end{aligned}
$$

Moreover, by the Gronwall inequality we infer that

$$
\int_{\Omega} \lambda^{2} d x \leq C r_{0} b_{0}^{2} e^{b_{0}^{2} t r_{0}^{-2}} \int_{t_{1}}^{t} \int_{\partial \Omega} h^{2} d \sigma d \tau .
$$

Finally, integrating (4.61) over the interval $\left[t_{1}, t\right]$ and combining the obtained inequality with (4.60) we get the desired estimate (4.56).

Proof of Proposition 3.4. In the sequel we shall maintain the notation $h_{i}=u_{i}^{2} \partial_{\nu} \lambda_{i}$ and we drop all the subindexes $i$. After straightforward computation we observe that

$$
\frac{\tilde{g}}{g}-1=\frac{u}{g} \partial_{\nu} \lambda+\lambda
$$

From this identity and by (2.4) we get

$$
\left\|\frac{\tilde{g}}{g}-1\right\|_{L^{2}\left(A^{\left.2 r_{0} \times\left[t_{1}, T\right]\right)}\right.} \leq \frac{1}{C_{0} b_{1}}\|h\|_{L^{2}\left(A \times\left[t_{1}, T\right]\right)}+\|\lambda\|_{L^{2}\left(\partial \Omega \times\left[t_{1}, T\right]\right)},
$$

where $C_{0}$ has been introduced in (2.4). Now, by integrating the trace estimate (4.59) over the time interval $\left[t_{1}, T\right]$ and by (4.56) we get

$$
\begin{aligned}
& \int_{t_{1}}^{T} \int_{\partial \Omega} \lambda^{2} d \sigma d \tau \leq C\left(r_{0}^{-1} \int_{t_{1}}^{T} \int_{\Omega} \lambda^{2} d x d \tau+r_{0} \int_{t_{1}}^{t} \int_{\Omega}|\nabla \lambda|^{2} d x d \tau\right) \\
\leq & C C_{0} r_{0}^{2}\left(1+e^{b_{0}^{2} r_{0}^{-2} t}\right)\left(1+b_{0}^{2} r_{0}^{-2} t\right) \int_{t_{1}}^{T} \int_{A} h^{2} d \sigma d \tau .
\end{aligned}
$$

At this stage we use the above inequality to control the right hand side of (4.62) obtaining the following:

$$
\left\|\frac{\tilde{g}}{g}-1\right\|_{L^{2}\left(A^{\left.2 r_{0} \times\left[t_{1}, T\right]\right)}\right.} \leq C_{1}\|h\|_{L^{2}\left(A \times\left[t_{1}, T\right]\right)},
$$


where $C_{1}=\left(\frac{1}{C_{0} b_{1}}+\left[C C_{0}\left(1+e^{b_{0}^{2} r_{0}^{-2} T}\right)\left(1+b_{0}^{2} r_{0}^{-2} T\right)\right]^{\frac{1}{2}}\right)$. Recalling that for every $c \in \mathbb{R}$ we have

$$
\left\|\frac{\tilde{g}}{g}-c\right\|_{L^{2}\left(A^{\left.2 r_{0} \times\left[t_{1}, T\right]\right)}\right.} \geq\left\|\frac{\tilde{g}}{g}-\left(\frac{\tilde{g}}{g}\right)_{A^{2 r_{0} \times[0, T]}}\right\|_{L^{2}\left(A^{\left.2 r_{0} \times[0, T]\right)}\right.} \geq \Phi_{0},
$$

from (4.64) we infer that

$$
\left\|\frac{\tilde{g}}{g}-\left(\frac{\tilde{g}}{g}\right)_{A^{2 r_{0} \times[0, T]}}\right\|_{L^{2}\left(A^{\left.2 r_{0} \times[0, T]\right)}\right.} \leq C_{1}\|h\|_{L^{2}\left(A \times\left[t_{1}, T\right]\right)} .
$$

At this point we claim that for any $\rho>0$ and for any $x_{0} \in \Omega_{\rho}$ it holds that

$$
\|h\|_{L^{2}\left(A \times\left[t_{1}, T\right]\right)}^{2} \leq \frac{C}{r_{0}^{n+2}} \int_{t_{1}}^{T} \int_{B_{\rho}\left(x_{0}\right)} \lambda^{2} d x d \tau,
$$

where $C>0$ is a constant depending on the a priori data and on $\rho$ only. Our claim, now, follows by standard arguments based on Theorem 3.7 and the corresponding version in the interior (see [24, Proposition 4.1.3]).

Remark 4.6. We observe that if, instead of problem (1.1), we consider the initialboundary value problem with the Robin condition on the whole lateral boundary $\partial \Omega \times[0, T]$, then the proof of the stability for the problem of determining the Robin coefficient on $I \times[0, T]$, assuming that it is known on $A \times[0, T]$, can be obtained by a slight change of the proof of Theorem 2.5.

More precisely, let $u$ be the solution to

$$
\begin{cases}u_{t}-\Delta u=0, & \text { in } \Omega \times[0, T], \\ u(x, 0)=0, & \text { in } \Omega, \\ \frac{\partial u}{\partial \nu}+\gamma(x, t) u=g(x, t), & \text { on } A \times[0, T], \\ \frac{\partial u}{\partial \nu}+\gamma(x, t) u=0, & \text { on } I \times[0, T] .\end{cases}
$$

Assume that $\Omega$ and $g(x, t)$ satisfy assumptions (2.1) and (2.2) respectively. Moreover, assume that $\gamma$ satisfies, instead of conditions (2.3), the conditions

$$
\gamma \in C^{0,1}(\partial \Omega \times[0, T])
$$

and

$$
0 \leq \gamma(x, t) \leq \bar{\gamma} r_{0}^{-1}
$$

Then the estimates (2.5), (2.6) and (2.7) of Theorem 2.5 continue to be true.

Indeed, it is a simple matter to check that an application of the Hopf lemma still implies (3.1) and, with an obvious interpretation of symbols, (3.3) and Propositions 3.1, 3.2, 3.4 are equally true.

Concerning Proposition 3.3, the following simple changes in the proof of (4.35) are sufficient. Let $y_{0} \in A_{2 r_{0}}$ and $t_{2}=\frac{t_{1}}{2}$; by the boundary condition and (4.67) we get

$$
\frac{\partial u}{\partial \nu}\left(y_{0}, t_{2}\right)=g\left(y_{0}, t_{2}\right)-\gamma\left(y_{0}, t_{2}\right) u\left(y_{0}, t_{2}\right) \geq \Phi_{1} r_{0}^{-1}-\bar{\gamma} r_{0}^{-1} u\left(y_{0}, t_{2}\right)
$$


Now, two cases occur: either

$$
u\left(y_{0}, t_{2}\right) \leq \frac{\Phi_{1}}{2 \bar{\gamma}}
$$

or

$$
u\left(y_{0}, t_{2}\right) \geq \frac{\Phi_{1}}{2 \bar{\gamma}}
$$

If (4.69) occurs, then

$$
\frac{\partial u}{\partial \nu}\left(y_{0}, t_{2}\right) \geq \frac{\Phi_{1} r_{0}^{-1}}{2 \bar{\gamma}},
$$

and (4.35) follows along the same lines as the proof of Proposition 3.3 .

If case (4.70) occurs, then by the Lagrange theorem and inequality (2.4) we have

$$
u\left(y_{0}-\tau_{0} \nu, t_{2}\right) \geq \frac{\Phi_{1}}{4 \bar{\gamma}},
$$

where $\tau_{0}=r_{0} \min \left\{\frac{\Phi_{1}}{4 \bar{\gamma} E}, 1\right\}$ and, again (4.35) is proved.

Under conditions (4.66) and (4.67) the second part (i.e. the $1^{\text {st }}$ step and the $2^{\text {nd }}$ step) of the proof of Proposition 3.3 remains unchanged.

\section{Proof of Proposition 4.3 \\ (HARNACK INEQUALITY AT THE BOUNDARY)}

Proof. Owing to the boundary regularity of $\Omega$, the set $B_{r_{0}} \cap \Omega$ is such that

$$
B_{r_{0}} \cap \Omega \equiv\left\{x \in \mathbb{R}^{n}: x_{n}>\varphi\left(x^{\prime}\right)\right\},
$$

where $\varphi \in C^{1, \alpha}\left(B_{r_{0}}^{\prime}\right)$ satisfies

$$
\varphi(0)=|\nabla \varphi(0)|=0, \quad\|\varphi\|_{C^{1, \alpha}\left(B_{r_{0}}^{\prime}\right)} \leq L r_{0} .
$$

We set $B_{\rho}^{+}=\left\{|x|<\rho, x_{n} \geq 0\right\}$.

We define the map $x=\Psi(z), \Psi \in C^{1, \alpha}\left(B_{r_{2}}\right)$ such that

$$
\begin{gathered}
\Psi\left(B_{r_{2}}\right) \subset B_{r_{1}}, \\
\Psi(z):=\left(z^{\prime}, \varphi\left(z^{\prime}\right)+z_{n}\right), \\
\Psi\left(B_{r_{2}}^{+}\right) \subset B_{r_{1}} \cap \Omega, \\
\frac{1}{2}\left|z_{1}-z_{2}\right| \leq\left|\Psi\left(z_{1}\right)-\Psi\left(z_{2}\right)\right| \leq C_{2}\left|z_{1}-z_{2}\right|, \quad \forall z_{1}, z_{2} \in B_{r_{2}}, \\
\frac{1}{2^{n}} \leq|\operatorname{det} D \Psi| \leq C_{3},
\end{gathered}
$$

where $r_{i}=\theta_{i} r_{0}, 0<\theta_{i}<1, i=1,2$, and $C_{2}, C_{3}, \theta_{1}, \theta_{2}$ only depend on $L$.

Denoting

$$
\widehat{A}(z, t)=|\operatorname{det} D \Psi|\left(D \Psi^{-1}\right)(\Psi(z)) A(\Psi(z), t)\left(D \Psi^{-1}\right)^{T}(\Psi(z)),
$$

we have

$$
\begin{gathered}
\frac{\lambda^{-1}}{2^{n+1}}|\xi|^{2} \leq \widehat{A}(z, t) \xi \cdot \xi \leq C_{4}|\xi|^{2}, \quad \forall z \in B_{r_{2}}^{+}, \forall t \in[0, T], \forall \xi \in \mathbb{R}^{n}, \\
\left|\widehat{A}\left(z_{1}, t\right)-\widehat{A}\left(z_{2}, t\right)\right| \leq \frac{C_{5}}{r_{0}}\left|z_{1}-z_{2}\right|, \quad \forall z_{1}, z_{2} \in B_{r_{2}}^{+}, \forall t \in[0, T],
\end{gathered}
$$

where $C_{4}, C_{5}$ only depend on $\lambda, L$. 
Denoting by $\widehat{\gamma}(z, t):=\gamma(\Psi(z), t)$, the function $\widehat{u}(z, t):=u(\Psi(z), t)$ satisfies the problem

$$
\begin{cases}\operatorname{div}(\widehat{A}(z, t) \nabla \widehat{u})-\partial_{t} \widehat{u}=0, & B_{r_{2}}^{+} \times(0, T], \\ \widehat{A}(z, t) \nabla \widehat{u} \cdot \nu+\widehat{\gamma}(z, t) \widehat{u}=0, & \left(|z|<r_{2}, z_{n}=0\right) \times(0, T],\end{cases}
$$

where $\nu=(0, \ldots, 0,-1)$.

Let $T_{1} \in(0, T)$ and

$$
0<r \leq \min \left\{\bar{\theta} r_{0}, \sqrt{T-T_{1}}, \sqrt{T_{1}}\right\},
$$

where $\bar{\theta}=\frac{\theta_{2}}{2}$.

Define

$$
\begin{aligned}
v(y, s) & :=\widehat{u}\left(r y, r^{2} s\right), \\
\widetilde{A}(y, s) & :=\widehat{A}\left(r y, r^{2} s\right), \\
\widetilde{\gamma}(y, s) & :=\widehat{\gamma}\left(r y, r^{2} s\right) .
\end{aligned}
$$

Then $v(y, s)$ satisfies the problem

$$
\begin{cases}\operatorname{div}(\widetilde{A}(y, s) \nabla v)-\partial_{s} v=0, & B_{\frac{r_{2}}{r}}^{+} \times\left(0, \frac{T}{r^{2}}\right], \\ \widetilde{A}(y, s) \nabla v \cdot \nu+r \widetilde{\gamma}(y, s) v=0, & \left(|y|<\frac{r_{2}}{r}, y_{n}=0\right) \times\left(0, \frac{T}{r^{2}}\right] .\end{cases}
$$

Since $\frac{r_{2}}{r} \geq 2$ and $\frac{T}{r^{2}} \geq \frac{T_{1}}{r^{2}}+1, v(y, s)$ also satisfies

$$
\begin{cases}\operatorname{div}(\widetilde{A}(y, s) \nabla v)-\partial_{s} v=0, & B_{1}^{+} \times\left(0, \frac{T_{1}}{r^{2}}+1\right], \\ \widetilde{A}(y, s) \nabla v \cdot \nu+r \widetilde{\gamma}(y, s) v=0, & \widetilde{\Gamma} \times\left(0, \frac{T_{1}}{r^{2}}+1\right],\end{cases}
$$

where $\widetilde{\Gamma}=\left(|y|<1, y_{n}=0\right)$.

Now we state two lemmas that adapt to the solutions of parabolic equations with Robin boundary condition the Lemmas 1, 2 of J. Moser [20].

Notation to Lemmas 5.1, 5.2.

$\sigma(x, t) \in L^{\infty}\left(\mathbb{R}^{n+1}\right)$ a symmetric matrix such that

$$
\lambda^{-1}|\xi|^{2} \leq \sigma(x, t) \xi \cdot \xi \leq \lambda|\xi|^{2}, \quad \forall(x, t) \in \mathbb{R}^{n+1}, \forall \xi \in \mathbb{R}^{n},
$$

$S(\rho)=\left\{|x|<\rho, x_{n}>0 ;|t|<\rho^{2}\right\}$,

$S^{+}(\rho)=\left\{|x|<\rho, x_{n}>0 ; 0<t<\rho^{2}\right\}$,

$S^{-}(\rho)=\left\{|x|<\rho, x_{n}>0 ;-\rho^{2}<t<0\right\}$.

Lemma 5.1. Let $u(x, t)>0$ be a solution of the problem

$$
\begin{cases}\operatorname{div}(\sigma(x, t) \nabla u(x, t))=u_{t}(x, t), & B_{2}^{+} \times(-2,2), \\ \sigma(x, t) \nabla u(x, t) \cdot \nu(x)+\gamma_{0}(x, t) u(x, t)=0, & \left(|x|<2, x_{n}=0\right) \times(-2,2) .\end{cases}
$$

Let $\left\|\gamma_{0}\right\|_{L^{\infty}\left(\left(|x|<2, x_{n}=0\right) \times(-2,2)\right)} \leq \bar{\gamma}_{0}$. Let $\frac{1}{2} \leq \rho<r \leq 1$; then there exists a constant $C_{6}$, depending on $\bar{\gamma}_{0}, n$, such that

$$
\begin{aligned}
& \sup _{S(\rho)} u^{p} \leq \frac{C_{6}}{(r-\rho)^{n+2}} \iint_{S(r)} u^{p} d x d t, \quad \forall p, \quad 0<p<\frac{1}{2 \lambda}, \\
& \sup _{S^{-}(\rho)} u^{p} \leq \frac{C_{6}}{(r-\rho)^{n+2}} \iint_{S^{-}(r)} u^{p} d x d t, \quad \forall p, \quad-\frac{1}{2 \lambda}<p<0 .
\end{aligned}
$$


Proof. Let $\Phi(x, t)$ be a test function such that

$$
\left\{\begin{array}{l}
\Phi \in C^{1}(|x| \leq 1,|t| \leq 1) \\
\Phi=0, \text { for }|x|=1,|t| \leq 1
\end{array}\right.
$$

By (5.5), integrating over $B_{1}^{+} \times\left(t_{1}, t_{2}\right),-1 \leq t_{1}<t_{2} \leq 1$, taking into account the Robin condition and denoting by $I(\rho)=\left\{|x|<\rho, x_{n}=0\right\}$, we get

$$
\begin{aligned}
& \int_{t_{1}}^{t_{2}} \int_{B_{1}^{+}} u_{t} \Phi+\int_{t_{1}}^{t_{2}} \int_{B_{1}^{+}} \sigma \nabla u \cdot \nabla \Phi=\int_{t_{1}}^{t_{2}} \int_{B_{1}^{+}} \sigma \nabla u \cdot \nu \Phi \\
& =-\int_{t_{1}}^{t_{2}} \int_{I(1)} \gamma_{0}(x, t) u \Phi .
\end{aligned}
$$

Let us set $v=u^{\frac{p}{2}}, \Phi=u^{p-1} \psi^{2}$; then we obtain

$$
\begin{aligned}
& \int_{t_{1}}^{t_{2}} \int_{B_{1}^{+}} \frac{2}{p} v v_{t} \psi^{2}+\int_{t_{1}}^{t_{2}} \int_{B_{1}^{+}} \frac{4(p-1)}{p^{2}} \sigma \nabla v \cdot \nabla v \psi^{2}+\int_{t_{1}}^{t_{2}} \int_{B_{1}^{+}} \frac{4}{p} v \psi \sigma \nabla v \cdot \nabla \psi \\
& =-\int_{t_{1}}^{t_{2}} \int_{I(1)} \gamma_{0} v^{2} \psi^{2} .
\end{aligned}
$$

Multiplying for $\frac{p}{4}$ and adding to both ends $\int_{t_{1}}^{t_{2}} \int_{B^{+}(1)} \frac{1}{4}\left(\frac{d}{d t} \psi^{2}\right) v^{2}$, we get

$$
\begin{aligned}
& \frac{1}{4} \int_{t_{1}}^{t_{2}} \int_{B_{1}^{+}} \frac{d}{d t}\left(\psi^{2} v^{2}\right)+\left(1-\frac{1}{p}\right) \int_{t_{1}}^{t_{2}} \int_{B_{1}^{+}} \sigma \nabla v \cdot \nabla v \psi^{2} \\
& =-\int_{t_{1}}^{t_{2}} \int_{B_{1}^{+}} v \psi \sigma \nabla v \cdot \nabla \psi+\frac{1}{2} \int_{t_{1}}^{t_{2}} \int_{B_{1}^{+}} \psi \psi_{t} v^{2}-\frac{p}{4} \int_{t_{1}}^{t_{2}} \int_{I(1)} \gamma_{0} v^{2} \psi^{2} .
\end{aligned}
$$

The term $\int_{t_{1}}^{t_{2}} \int_{I(1)} \gamma_{0} v^{2} \psi^{2}$ may be estimated by the trace theorem [1, Theorem 5.22 ] and Poincaré inequality (since $\psi=0$ for $|x|=1, x_{n}>0,|t| \leq 1$ ); that is,

$$
\left|\int_{t_{1}}^{t_{2}} \int_{I(1)} \gamma_{0} v^{2} \psi^{2}\right| \leq C \bar{\gamma}_{0} \int_{t_{1}}^{t_{2}} \int_{B_{1}^{+}}\left|\nabla\left(v^{2} \psi^{2}\right)\right|,
$$

where $C$ is an absolute constant.

Then, by simple estimates, we get

$$
\begin{aligned}
& \left|\frac{p}{4} \int_{t_{1}}^{t_{2}} \int_{I(1)} \gamma_{0} v^{2} \psi^{2}\right| \leq C \bar{\gamma}_{0} \frac{|p|}{4} \int_{t_{1}}^{t_{2}} \int_{B_{1}^{+}}\left|\nabla\left(v^{2} \psi^{2}\right)\right| \leq \widetilde{\varepsilon} \int_{t_{1}}^{t_{2}} \int_{B_{1}^{+}}|\nabla v|^{2} \psi^{2} \\
& +\frac{1}{\widetilde{\varepsilon}} \frac{C^{2} \bar{\gamma}_{0}^{2}|p|^{2}}{16} \int_{t_{1}}^{t_{2}} \int_{B_{1}^{+}} v^{2} \psi^{2}+\frac{C \bar{\gamma}_{0}|p|}{4} \int_{t_{1}}^{t_{2}} \int_{B_{1}^{+}} v^{2}\left(\psi^{2}+|\nabla \psi|^{2}\right) .
\end{aligned}
$$

We consider first the case $\left(1-\frac{1}{p}\right)>0$.

By (5.6), (5.8) and by

$$
|v \psi \sigma \nabla v \cdot \nabla \psi| \leq \frac{1}{4 \widetilde{\varepsilon}} v^{2} \nabla \psi \cdot \sigma \nabla \psi+\widetilde{\varepsilon} \psi^{2} \nabla v \cdot \sigma \nabla v,
$$

we obtain, taking $\widetilde{\varepsilon}=\frac{1}{\lambda+1} \frac{1}{2}\left(1-\frac{1}{p}\right)$,

$$
\begin{aligned}
& \frac{1}{4} \int_{t_{1}}^{t_{2}} \int_{B_{1}^{+}} \frac{d}{d t}\left(v^{2} \psi^{2}\right)+\frac{1}{2 \lambda}\left(1-\frac{1}{p}\right) \int_{t_{1}}^{t_{2}} \int_{B_{1}^{+}}|\nabla v|^{2} \psi^{2} \\
& \leq \frac{\lambda(\lambda+1)}{2} \frac{p}{p-1} \int_{t_{1}}^{t_{2}} \int_{B_{1}^{+}} v^{2}|\nabla \psi|^{2}+\frac{1}{2} \int_{t_{1}}^{t_{2}} \int_{B_{1}^{+}} v^{2}\left|\psi \psi_{t}\right| \\
& +\frac{C^{2} \bar{\gamma}_{0}^{2}}{8}(\lambda+1) \frac{p^{3}}{p-1} \int_{t_{1}}^{t_{2}} \int_{B_{1}^{+}} v^{2} \psi^{2}+\frac{C \bar{\gamma}_{0}|p|}{4} \int_{t_{1}}^{t_{2}} \int_{B_{1}^{+}} v^{2}\left(\psi^{2}+|\nabla \psi|^{2}\right) .
\end{aligned}
$$


Collecting and estimating the last two terms we obtain

$$
\begin{aligned}
& \frac{1}{4} \int_{t_{1}}^{t_{2}} \int_{B^{+}(1)} \frac{d}{d t}\left(v^{2} \psi^{2}\right)+\frac{1}{2 \lambda}\left(1-\frac{1}{p}\right) \int_{t_{1}}^{t_{2}} \int_{B^{+}(1)}|\nabla v|^{2} \psi^{2} \\
& \leq \frac{1}{2} \lambda(\lambda+1) \frac{p}{p-1} \int_{t_{1}}^{t_{2}} \int_{B^{+}(1)} v^{2}|\nabla \psi|^{2}+\frac{1}{2} \int_{t_{1}}^{t_{2}} \int_{B^{+}(1)} v^{2}\left|\psi \psi_{t}\right| \\
& +C\left(\lambda, \bar{\gamma}_{0}\right)\left(\frac{p^{3}}{p-1}+|p|\right) \int_{t_{1}}^{t_{2}} \int_{B^{+}(1)} v^{2}\left(\psi^{2}+|\nabla \psi|^{2}\right) .
\end{aligned}
$$

In the case $\left(1-\frac{1}{p}\right)<0$, we multiply relation (5.6) by -1 ; by (5.8), (5.9), choosing $\widetilde{\varepsilon}=\frac{1}{\lambda+1} \frac{1}{2}\left(\frac{1}{p}-1\right)$, we obtain

$$
\begin{aligned}
& -\frac{1}{4} \int_{t_{1}}^{t_{2}} \int_{B_{1}^{+}} \frac{d}{d t}\left(v^{2} \psi^{2}\right)+\frac{1}{2 \lambda}\left(\frac{1}{p}-1\right) \int_{t_{1}}^{t_{2}} \int_{B_{1}^{+}}|\nabla v|^{2} \psi^{2} \\
& \leq \frac{\lambda(\lambda+1)}{2} \frac{p}{1-p} \int_{t_{1}}^{t_{2}} \int_{B_{1}^{+}} v^{2}|\nabla \psi|^{2}+\frac{1}{2} \int_{t_{1}}^{t_{2}} \int_{B_{1}^{+}} v^{2}\left|\psi \psi_{t}\right| \\
& +C\left(\lambda, \bar{\gamma}_{0}\right)\left(\frac{p^{3}}{1-p}+|p|\right) \int_{t_{1}}^{t_{2}} \int_{B_{1}^{+}} v^{2}\left(\psi^{2}+|\nabla \psi|^{2}\right) .
\end{aligned}
$$

(5.11), (5.12) are analogous to the inequality in [20], page 737, with the addition of the term $C\left(\lambda, \bar{\gamma}_{0}\right)\left(\frac{p^{3}}{1-p}+|p|\right) \int_{t_{1}}^{t_{2}} \int_{B_{1}^{+}} v^{2}\left(\psi^{2}+|\nabla \psi|^{2}\right)$. Proceeding in the same way, we get the thesis of Lemma 5.1.

Lemma 5.2. Let $u(x, t)>0$ be a solution of the problem

$$
\begin{cases}\operatorname{div}(\sigma(x, t) \nabla u(x, t))=u_{t}(x, t), & B_{2}^{+} \times(-2,2), \\ \sigma(x, t) \nabla u(x, t) \cdot \nu(x)+\gamma_{0}(x, t) u(x, t)=0, & \left(|x|<2 \cap x_{n}=0\right) \times(-2,2) .\end{cases}
$$

Let $\left\|\gamma_{0}\right\|_{L^{\infty}\left(\left(|x|<2, x_{n}=0\right) \times(-2,2)\right)} \leq \bar{\gamma}_{0}$. Then there exist constants a, $C_{7}$ such that meas $\left\{(x, t) \in S^{+}(1): \log u<-s+a\right\}+$ meas $\left\{(x, t) \in S^{-}(1): \log u>s+a\right\} \leq \frac{C_{7}}{s}$, for every $s>0$, where $C_{7}$ depends on $\left(\lambda, n, \bar{\gamma}_{0}\right)$, while a depends on $u$.

Proof. We consider the function

$$
v=-\log u
$$

that satisfies the problem

$$
\begin{cases}v_{t}-\operatorname{div}(\sigma \nabla v)=-\nabla v \cdot \sigma \nabla v, & B_{2}^{+} \times(-2,2), \\ \sigma \nabla v \cdot \nu=\gamma_{0}, & \left(|x|<2 \cap x_{n}=0\right) \times(-2,2) .\end{cases}
$$

Let $\psi^{2}(x)$ be a test function independent of $t$ and such that $\psi(x) \geq 0, \psi(x)=0$ for $|x|=2, x_{n}>0$. By (5.13) , we get, adding the null term $v \frac{d}{d t} \psi^{2}$,

$$
\begin{gathered}
\int_{t_{1}}^{t_{2}} \int_{B_{2}^{+}} \frac{d}{d t}\left(v \psi^{2}\right)+2 \int_{t_{1}}^{t_{2}} \int_{B_{2}^{+}} \sigma \nabla v \cdot \nabla \psi \psi \\
-\int_{t_{1}}^{t_{2}} \int_{I(2)} \gamma_{0} \psi^{2}+\int_{t_{1}}^{t_{2}} \int_{B_{2}^{+}} \sigma \nabla v \cdot \nabla v \psi^{2}=0 .
\end{gathered}
$$

By Schwartz inequality, since

$$
\int_{t_{1}}^{t_{2}} \int_{B_{2}^{+}} \sigma \nabla v \cdot \nabla \psi \psi \geq-\frac{1}{4} \int_{t_{1}}^{t_{2}} \int_{B_{2}^{+}} \sigma \nabla v \cdot \nabla v \psi^{2}-\int_{t_{1}}^{t_{2}} \int_{B_{2}^{+}} \sigma \nabla \psi \cdot \nabla \psi,
$$


we get, by (5.14),

$$
\left.\int_{B_{2}^{+}} v \psi^{2}\right|_{t_{1}} ^{t_{2}}+\frac{1}{2 \lambda} \int_{t_{1}}^{t_{2}} \int_{B_{2}^{+}}|\nabla v|^{2} \psi^{2} \leq 2 \int_{t_{1}}^{t_{2}} \int_{B_{2}^{+}} \sigma \nabla \psi \cdot \nabla \psi+\int_{t_{1}}^{t_{2}} \int_{I(2)} \gamma_{0} \psi^{2} .
$$

Again by the trace theorem [1, Theorem 5.22] and Poincaré inequality we finally obtain

$$
\begin{gathered}
\left.\int_{B_{2}^{+}} v \psi^{2}\right|_{t_{1}} ^{t_{2}}+\frac{1}{2 \lambda} \int_{t_{1}}^{t_{2}} \int_{B_{2}^{+}}|\nabla v|^{2} \psi^{2} \leq 2 \int_{t_{1}}^{t_{2}} \int_{B_{2}^{+}} \sigma \nabla \psi \cdot \nabla \psi \\
+C \bar{\gamma}_{0} \int_{t_{1}}^{t_{2}} \int_{B_{2}^{+}}\left(\psi^{2}+|\nabla \psi|^{2}\right) \leq C\left(\bar{\gamma}_{0}, \lambda\right) \int_{t_{1}}^{t_{2}} \int_{B_{2}^{+}}\left(\psi^{2}+|\nabla \psi|^{2}\right) .
\end{gathered}
$$

(5.15) is analogous to the inequality in [1], page 121, with the addition of the term $C\left(\bar{\gamma}_{0}, \lambda\right) \int_{t_{1}}^{t_{2}} \int_{B_{2}^{+}}\left(\psi^{2}+|\nabla \psi|^{2}\right)$. Proceeding in the same way, we get the thesis of Lemma 5.2.

Conclusion of the proof of Proposition 4.2. By Lemma 3 in [20, following the same lines as the proof of statement (5) in [20, page 734], we get the result

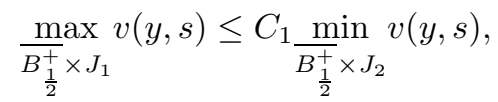

where $J_{1}=\left[\frac{T_{1}}{r^{2}}, \frac{T_{1}}{r^{2}}+\frac{1}{3}\right], J_{2}=\left[\frac{T_{1}}{r^{2}}+\frac{2}{3}, \frac{T_{1}}{r^{2}}+1\right]$, and where, since

$$
\|r \widetilde{\gamma}(y, s)\|_{L^{\infty}\left(\widetilde{\Gamma} \times\left(0, \frac{T_{1}}{r^{2}}+1\right]\right)} \leq r \frac{\bar{\gamma}}{r_{0}} \leq \bar{\gamma},
$$

the constant $C_{1}$ depends on $\lambda, \bar{\gamma}, L$ and $n$.

Now, returning back to the plane $(z, t)$, we have for the function $\widehat{u}(z, t)$

$$
\max _{\overline{B_{\frac{r}{2}}^{+}} \times\left[T_{1}, T_{1}+\frac{r^{2}}{3}\right]} \widehat{u}(z, t) \leq C_{1} \underset{B_{\frac{r}{2}}^{+} \times\left[T_{1}+\frac{2 r^{2}}{3}, T_{1}+r^{2}\right]}{ } \widehat{u}(z, t),
$$

where $C_{1}$ depends on $\lambda, \bar{\gamma}, L$ and $n$.

At last we return to the plane $(x, t)$, that is, to the function $u(x, t)$. We use for this purpose the relation

$$
\Omega \cap B_{\frac{\beta}{2}} \subset \Psi\left(B_{\beta}^{+}\right) \subset \Omega \cap B_{C_{2} \beta}, \quad \forall \beta \leq r_{2} .
$$

We choose $\beta=\frac{r}{2}$; then, by the above left inclusion, we get

$$
\frac{\max }{\left(|x|<\frac{r}{4}\right) \cap \Omega \times\left[T_{1}, T_{1}+\frac{r^{2}}{3}\right]} u(x, t) \leq \max _{\left(|z| \leq \frac{r}{2}, z_{n} \geq 0\right) \times\left[T_{1}, T_{1}+\frac{r^{2}}{3}\right]} \widehat{u}(z, t)
$$

and

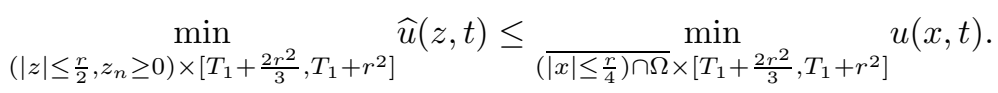

By (5.17), (5.18), (5.19), we obtain the final thesis,

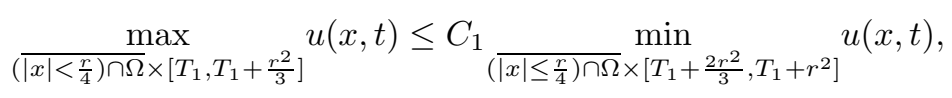

where $C_{1}$ depends on $\lambda, \bar{\gamma}, L$ and $n$. 


\section{REFERENCES}

[1] Robert A. Adams, Sobolev spaces, Academic Press [A subsidiary of Harcourt Brace Jovanovich, Publishers], New York-London, 1975. Pure and Applied Mathematics, Vol. 65. MR.0450957 (56 \#9247)

[2] Giovanni Alessandrini, Elena Beretta, Edi Rosset, and Sergio Vessella, Optimal stability for inverse elliptic boundary value problems with unknown boundaries, Ann. Scuola Norm. Sup. Pisa Cl. Sci. (4) 29 (2000), no. 4, 755-806. MR1822407 (2002b:35196)

[3] Valeria Bacchelli, Uniqueness for the determination of unknown boundary and impedance with the homogeneous Robin condition, Inverse Problems 25 (2009), no. 1, 015004, 4, DOI 10.1088/0266-5611/25/1/015004. MR2465336(2009m:35531)

[4] E. Beretta and S. Vessella, Stable determination of boundaries from Cauchy data, SIAM J. Math. Anal. 30 (1999), no. 1, 220-232, DOI 10.1137/S0036141097325733. MR.1656995 (99j:35229)

[5] P. Bison, M. Ceseri, D. Fasino, and G. Inglese, Active infrared thermography in nondestructive evaluation of surface corrosion. II. Heat exchange between specimen and environment, Applied and industrial mathematics in Italy II, Ser. Adv. Math. Appl. Sci., vol. 75, World Sci. Publ., Hackensack, NJ, 2007, pp. 161-171, DOI 10.1142/9789812709394_0015. MR2367569

[6] P. Bison, M. Ceseri, D. Fasino, and G. Inglese, Domain derivative approach to active infrared thermography, Inverse Probl. Sci. Eng. 18 (2010), no. 7, 873-889, DOI 10.1080/17415977.2010.492511. MR2743228(2011i:35252)

[7] Fioralba Cakoni and Rainer Kress, Integral equations for inverse problems in corrosion detection from partial Cauchy data, Inverse Probl. Imaging 1 (2007), no. 2, 229-245, DOI 10.3934/ipi.2007.1.229. MR2282267 (2008j:35181)

[8] B. Canuto, E. Rosset, and S. Vessella, Quantitative estimates of unique continuation for parabolic equations and inverse initial-boundary value problems with unknown boundaries, Trans. Amer. Math. Soc. 354 (2002), no. 2, 491-535, DOI 10.1090/S0002-9947-01-02860-4. MR.1862557(2002k:35311)

[9] Michele Di Cristo and Luca Rondi, Examples of exponential instability for inverse inclusion and scattering problems, Inverse Problems 19 (2003), no. 3, 685-701, DOI 10.1088/02665611/19/3/313. MR.1984884(2004d:35247)

[10] Michele Di Cristo, Luca Rondi, and Sergio Vessella, Stability properties of an inverse parabolic problem with unknown boundaries, Ann. Mat. Pura Appl. (4) 185 (2006), no. 2, 223-255, DOI 10.1007/s10231-005-0152-x. MR2214134(2006m:35378)

[11] Luis Escauriaza and Francisco Javier Fernández, Unique continuation for parabolic operators, Ark. Mat. 41 (2003), no. 1, 35-60, DOI 10.1007/BF02384566. MR1971939(2004b:35136)

[12] David Gilbarg and Neil S. Trudinger, Elliptic partial differential equations of second order, Classics in Mathematics, Springer-Verlag, Berlin, 2001. Reprint of the 1998 edition. MR.1814364 (2001k:35004)

[13] Gabriele Inglese, An inverse problem in corrosion detection, Inverse Problems 13 (1997), no. 4, 977-994, DOI 10.1088/0266-5611/13/4/006. MR 1463588

[14] Victor Isakov, On uniqueness of obstacles and boundary conditions from restricted dynamical and scattering data, Inverse Probl. Imaging 2 (2008), no. 1, 151-165, DOI 10.3934/ipi.2008.2.151. MR2375327(2009h:35444)

[15] O. A. Ladyženskaja, V. A. Solonnikov, and N. N. Ural'ceva, Linear and quasilinear equations of parabolic type (Russian), Translated from the Russian by S. Smith. Translations of Mathematical Monographs, Vol. 23, American Mathematical Society, Providence, R.I., 1968. MR.0241822(39 \#3159b)

[16] Gary M. Lieberman, Regularized distance and its applications, Pacific J. Math. 117 (1985), no. 2, 329-352. MR779924 (87j:35101)

[17] Gary M. Lieberman, Second order parabolic differential equations, World Scientific Publishing Co. Inc., River Edge, NJ, 1996. MR.1465184 (98k:35003)

[18] J.-L. Lions and E. Magenes, Non-homogeneous boundary value problems and applications. Vol. II, Springer-Verlag, New York, 1972. Translated from the French by P. Kenneth; Die Grundlehren der mathematischen Wissenschaften, Band 182. MR0350178 (50 \#2671)

[19] Jürgen Moser, A Harnack inequality for parabolic differential equations, Comm. Pure Appl. Math. 17 (1964), 101-134. MR0159139 (28 \#2357) 
[20] J. Moser, On a pointwise estimate for parabolic differential equations, Comm. Pure Appl. Math. 24 (1971), 727-740. MR0288405 (44 \#5603)

[21] Carlo Domenico Pagani and Dario Pierotti, Identifiability problems of defects with the Robin condition, Inverse Problems 25 (2009), no. 5, 055007, 12, DOI 10.1088/02665611/25/5/055007. MR2501025 (2010k:35539)

[22] Eva Sincich, Stability for the determination of unknown boundary and impedance with a Robin boundary condition, SIAM J. Math. Anal. 42 (2010), no. 6, 2922-2943, DOI 10.1137/100788343. MR2745797 (2011m:35425)

[23] Sergio Vessella, Stability estimates in an inverse problem for a three-dimensional heat equation, SIAM J. Math. Anal. 28 (1997), no. 6, 1354-1370, DOI 10.1137/S0036141095294262. MR.1474218(99d:35178)

[24] Sergio Vessella, Quantitative estimates of unique continuation for parabolic equations, determination of unknown time-varying boundaries and optimal stability estimates, Inverse Problems 24 (2008), no. 2, 023001, 81, DOI 10.1088/0266-5611/24/2/023001. MR2408537 (2009c:35192)

[25] M. Vollmer, K. P. Möllmann, Infrared thermal imaging: fundamental, research and applications, Wiley-VCH Verlag GmbH \& Co KGaA, Weinheim, 2010.

Department of Mathematics, Politecnico di Milano, 20100 Milan, Italy

E-mail address: valeria.bacchelli@polimi.it

Department of Mathematics, Politecnico di Milano, 20100 Milan, Italy

E-mail address: michele.dicristo@polimi.it

Department of Mathematics, Università di Trieste, 34014 Trieste, Italy

E-mail address: esincich@units.it

Current address: Laboratory for Multiphase Processes, University of Nova Gorica, Vipavska

13, SI-5000 Nova Gorica, Slovenia

E-mail address: eva.sincich@ung.si

Department of Mathematics, Università di Firenze, 50121 Florence, Italy

E-mail address: sergio.vessella@unifi.it 